\title{
La simulation numérique discrète du comportement des matériaux granulaires
}

A. MAHBOUBI

A. GHAOUTI

B. CAMBOU

LTDS URA 855

Ecole Centrale de Lyon

Réseau GEO, BP 163.

69131 Ecully
Cet article a pour but de présenter la méthode numérique des éléments discrets qui permet de simuler le comportement des milieux granulaires en prenant en compte leur nature discrète. Après une présentation générale de la méthode deux types d'utilisation seront analysés.

Une première série d'essais homogènes bidimensionneis et tridimensionnels permet de mettre en évidence les phénomènes locaux qui sont à la base du comportement des milieux granulaires et constitue une aide puissante pour la compréhension fine du comportement de ces milieux et pour le développement de modèles de comportement continus.

Une deuxième série d'essais non-homogènes (vidange d'un silo) montre l'intérêt de l'approche numérique discrète dans le cas où la nature discontinue du milieu pilote les phénomènes à l'échelle globale. Pour ce type de problème une approche en terme de milieu continu apparaît peu crédible.

\section{The distinct numerical modelling of the behaviour of granular materials}

This paper presents the Distinct Element Method that permits the simulation of the behavior of granular materials taking into account their discrete state. After a general presentation, two types of use will be presented.

The first one performs two-dimensional and three-dimensional homogeneous tests permitting to point out the local phenomena which are the fundaments of the behavior of granular materials. The comprehension of these phenomena is a powerful help for understanding of their behavior and therefore for the development of constitutive models.

The second use consists to performe a non homogeneous test (discharge of a silo) to show the interest of the discrete numerical method when the discrete nature dominates the global behavior. In fact for this type of problem a continuum approach seems few credible. 


\section{Introduction}

Les matériaux granulaires sont constitués de particules qui se déplacent indépendamment l'une et l'autre et qui interagissent aux points de contact. Cette nature discrète rend le comportement mécanique de ces types de matériaux très complexe et, à ce jour, la définition d'une loi de comportement capable de représenter tous les aspects de leur comportement reste un problème ouvert.

Depuis une vingtaine d'années une méthode numérique a été développée pour modéliser le comportement de ce type de milieux. Cette méthode appelée la Méthode des Éléments Discrets (MED) traite un matériau granulaire comme un assemblage de particules en interaction au niveau des contacts qui peuvent être rompus ou formés à chaque pas de temps. La plupart des développements et des applications de la méthode des éléments discrets sont issus des travaux pionniers de Cundall $(1971,1974,1979)$, destinés à la modélisation bidimensionnelle, d'une part de milieux rocheux fracturés et, d'autre part, de milieux granulaires formés de cylindres. On notera aussi la méthode numérique originale développée par J.-J. Moreau $(1979,1988)$ et M. Jean et al. (1993).

La MED a permis au cours de ces dernières années de mettre en évidence et de mieux comprendre les phénomènes locaux (géométriques, cinématiques, statiques) qui sont à la base des phénomènes observés à l'échelle d'un échantillon. Elle a, en particulier, permis de mieux appréhender l'évolution de la structure interne du milieu au cours de la sollicitation, et a pu être utilisée comme aide au développement de modèles de comportement pour les sols granulaires (Bathurst et al., 1988).

Le but de cet article est de présenter les possibilités et les limitations de ce type de méthode ainsi que les résultats originaux développés dans notre laboratoíre. Les exemples traités dans cet article sont de deux ordres:

- des simulations d'essais en sollicitation homogène qui permettent d'analyser les phẻnomènes locaux et peuvent être utilisés comme une aide au développement de modèles constitutifs:
- des simulations d'essais non-homogènes ou la structure discrète du milieu est essentielle pour la modélisation du comportement global du milieu.

\section{2}

\section{Généralités sur la MED}

Nous présentons ici la MED sous la forme où elle a été développée dans le logiciel TRUBAL (Cundall, 1989) que nous avons utilisé pour les applications présentées dans cet article. La MED modélise un milieu granulaire par un assemblage de grains le plus souvent de forme simple (cylindres, sphères), le nombre des particules prises en compte dépend, évidemment, de la puissance informatique disponible, aujourd'hui la plupart des simulations réalisées prennent en compte entre 1000 et 10000 particules. Chaque particule est représentée par un élément discret (indépendant), le déplacement des particules est supposé être celui d'un corps rigide, les déformations étant supposées localisées aux points de contact (Fig. 1a). Le principe de la MED est simple, il est basé sur les applications répétitives de l'équation du mouvement d'un corps rigide soumis à une sollicitation (déplacement ou force) suivi par le calcul des forces engendrées par un contact entre deux particules (loi de contact). La figure 2 décrit un schéma simplifié de cette méthode.

Une fois que les forces pour tous les contacts entre les particules ont été calculées, les résultantes des forces et des moments sur chaque particule sont utilisées dans l'équation du mouvement pour déterminer la nouvelle position de chaque particule. La force et le moment obtenus pour le temps t sont supposés agir sur une particule pendant l'intervalle $\Delta t$ (de $t-\frac{1}{2} \Delta t$ jusqu'à $t+\frac{1}{2} \Delta t$ ).

Dans ce cas l'équation du mouvement de chaque particule s'écrit comme suit:

$$
m \ddot{x}+c \dot{x}-\Sigma F=m \ddot{x}+m \alpha \dot{x}-\Sigma F=0
$$

où $\mathrm{c}$ représente l'amortissement et $\mathrm{m}$ est la masse de la particule. F représente les forces extérieures et les forces de volume appliquées à la particule.

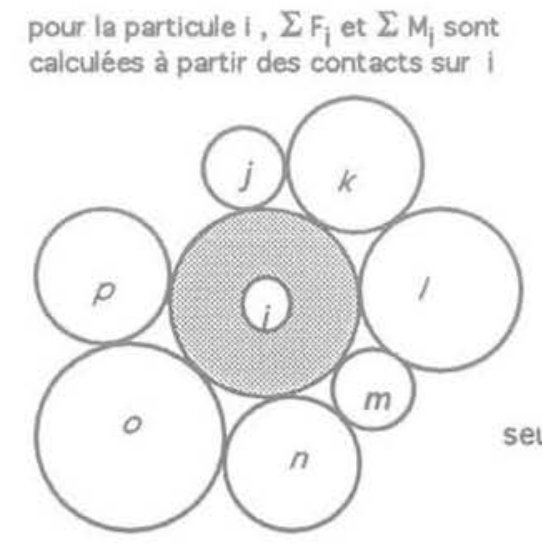

a)

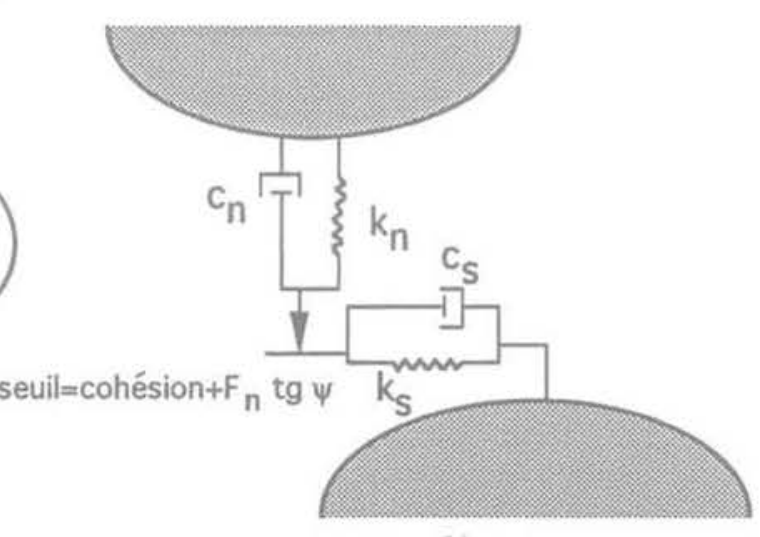

b)

FG. 1 Les notions de base, a) schéma d'équilibre local, b) mécanisme de contact.

Basic notions of distinct simulation, a) local equilibrium scheme, b) contact mechanical representation. 


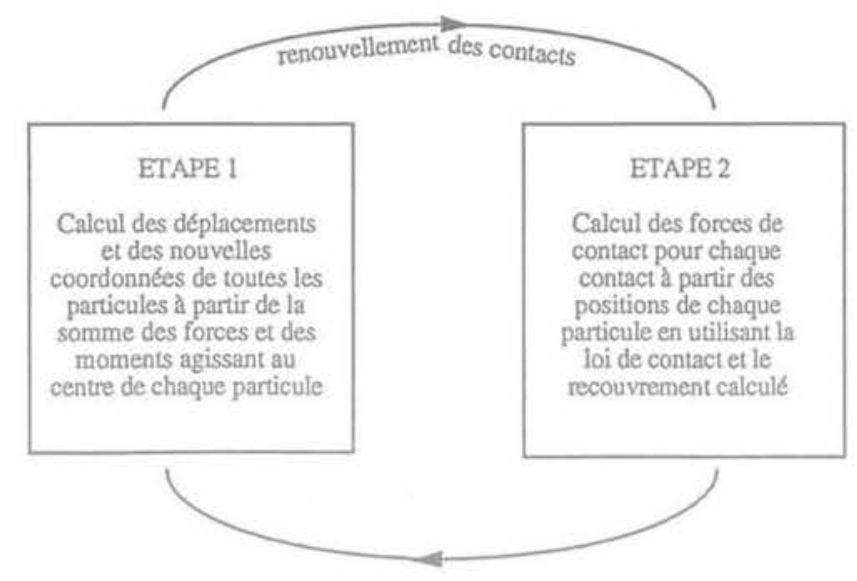

FIG. \& Schéma simple des deux étapes importantes de la MED.

Two important steps of the distinct element method.

L'intégration numérique par la méthode des différences finies conduit à:

$$
\begin{aligned}
\left(\dot{x}_{i}\right)_{1+\frac{1}{2}} & =\frac{\left(\dot{x}_{i}\right)_{i \frac{1}{2}}\left(1-\frac{\alpha \Delta t}{2}\right)+\frac{\Delta t}{m} \Sigma F_{t}}{\left(1+\frac{\alpha \Delta t}{2}\right)} \\
\left(\omega_{i}\right)_{1-\frac{1}{2}}= & \frac{\left(\omega_{i}\right)_{1 \frac{1}{2}}\left(1-\frac{\alpha \Delta t}{2}\right)+\frac{\Delta t}{1} \Sigma M_{1}}{\left(1+\frac{\alpha \Delta t}{2}\right)}
\end{aligned}
$$

Les nouvelles valeurs des coordonnées et rotations seront utilisées pour recalculer la nouvelle position et les nouvelles rotations de chacune des particules. L'intégration numérique de $\mathrm{x}$ et $\omega$ sur l'intervalle $\Delta t$ donne:

$$
\begin{aligned}
& \left(x_{i}\right)_{s+1}=\left(x_{i}\right)_{1}+\left(\dot{x}_{1}\right)_{n+\frac{1}{2}} \cdot \Delta t \\
& \left(\theta_{i}\right)_{t+1}=\left(\theta_{i}\right)_{1}+\left(\omega_{1}\right)_{n \in \frac{3}{2}} \cdot \Delta t
\end{aligned}
$$

Les relations (2) à (5) seront appliquées à toutes les particules pour calculer la nouvelle position et les nouvelles rotations. Ces nouvelles valeurs de position peuvent être, maintenant, utilisées dans la relation forcedéplacement relative au contact, et le cycle se répète pour un nouvel incrément de temps.

Du fait de la nature de l'algorithme, la convergence de la MED est conditionnelle. Elle dépend de l'amplitude de la propagation de la perturbation à chaque pas de temps, $\Delta t$. $\Delta$ t est supposé suffisamment petit de façon que, pour chaque $\Delta t$, la perturbation ne puisse se propager qu'aux particules voisines en contact. $\Delta$ t est en général défini à partir du temps critique minimal correspondant à un ensemble de deux particules en contact.

Généralement, un contact est modélisé par le modèle de Kelvin dans les directions normale et tangentielle avec un patin dans la direction tangentielle limitant la force tangentielle par un seuil (Fig. 1b). Les ressorts peuvent être linéaires ou non linéaires (par exemple, la loi non linéaire de Hertz, 1881). Le calcul des forces s'effectue par le biais de la loi de contact à partir du recouvrement des particules. On notera que la méthode proposée par Moreau et Jean ne prend pas en compte une véritable loi de contact, mais traite l'interaction entre particules sous la forme d'un choc élastique ou inélastique (M. Jean, 1993).

Si l'amortissement ou la vitesse de chargement est grand le comportement du milieu supposé sollicité de façon quasi statique peut être affecté par l'effet de l'inertie. On notera que l'amortissement n'a généralement pas un réel sens physique et est seulement utilisé pour obtenir la convergence numérique de calcul. Pour s'assurer que les forces d'inertie et les effets visqueux ne modifient pas le comportement réel du matériau certains tests sont effectués:

- un indice est défini comme le rapport entre la moyenne des résultantes des forces agissant sur chaque particule (non équilibrée) sur la moyenne des forces normales agissant dans le milieu. Cet indice ne doit pas dépasser un faible pourcentage (toujours moins de $1 \%$ );

- deux simulations peuvent être effectuées à deux vitesses de sollicitations différentes et les résultats doivent être identiques.

Finalement, les contraintes sont calculées à partir de la relation proposée par Love (1927):

$$
\sigma_{0}=\frac{1}{v} \Sigma\left(F_{1} \cdot 1_{1}\right)
$$

où, V est le volume de l'échantillon.

\section{3}

\section{Simulations d'essais biaxiaux homogènes (milieu bidimensionnel)}

L'assemblage est constitué de 1000 particules sphériques dont les centres sont localisés dans un plan et l'interaction entre les particules est limitée au contact physique, modélisé par la loi de Hertz-Mindlin (1953) avec frottement Coulombien et sans cohésion intergranulaire. L'échantillon est constitué de:

500 particules de diamètre $0,4 \mathrm{~mm}$.

300 particules de diamètre $0,5 \mathrm{~mm}$.

200 particules de diamètre $0,6 \mathrm{~mm}$.

Trois types de particules ont été pris en compte pour éviter d'avoir un échantillon monodisperse qui présente un comportement singulier, liẻ à la régularité des arrangements de particules (Cundall, 1989).

Le nombre total des particules a été choisi pour qu'il soit significatif d'un assemblage réel. Il semble que 1000 soit une limite inférieure pour une analyse bidimensionnelle (Kruyt, 1993). De nombreux chercheurs ont choisi ce mème nombre de particules (Cundall, 1988; Rothenburg et al., 1989; Thornton et al., 1986).

La distribution des particules choisie permet d'obtenir un échantillon avec un domaine de densité possible assez grand. Les caractéristiques mécaniques du matériau constitutif des particules apparaissent dans la loi de contact utilisée, le module de cisaillement, $\mathrm{G}=$ $29 \mathrm{GPa}$, et le coefficient de Poisson, $\mathrm{v}=0,2$ utilisés correspondent à celles du Quartz ( $\mathrm{Ng}$ et al., 1994), l'angle de frottement intergranulaire, $\psi$, est supposé égal à $26^{\circ}$ (sauf dans la phase initiale de compactage où il est réduit pour diminuer les temps de calcul). 
L'effet de la gravité n'a pas été considéré dans ces simulations. La mise en place des échantillons se fait à partir des étapes suivantes:

i) génération aléatoire des particules dans un carré périodique;

ii) compactage isotrope de l'échantillon en diminuant la surface de l'espace périodique de façon isotrope et avec un coefficient de frottement intergranulaire faible, $\tan \psi=0,05$. L'essai est poursuivi jusqu'à l'obtention d'un indice des vides e $=0,35$. La suite de la préparation des échantillons est alors différente pour les milieux dense et lấche;

iii) pour un échantillon dense, le compactage isotrope est poursuivi avec $\tan \psi=0,05$ jusqu'à l'obtention d'un indice des vides, e, égal à 0,197 et un contrainte isotrope de $550 \mathrm{kPa}\left(\sigma_{1}=\sigma_{2}=550 \mathrm{kPa}\right)$;

iv) pour un échantillon lâche, le compactage isotrope est poursuivi en considérant $\tan \psi=0,5$ jusqu'à l'obtention d'un indice des vides égal à 0,25 et une contrainte isotrope de $535 \mathrm{kPa}$.

Il y a lieu de remarquer que le compactage en présence d'un frottement relativement fort permet d'obtenir des échantillons lâches. A cette étape nous disposons de deux échantillons avec les densités souhaitées et bien équilibrés.

\section{1}

\section{Essais biaxiaux sur les échantillons dense et lâche}

L'essai biaxial est réalisé en compressant l'échantillon dans la direction 1 tout en gardant la contrainte dans la direction 2 constante. La sollicitation se fait par le biais d'un champ de déformation homogène. Le contrôle de la contrainte dans la direction latérale se fait par une boucle d'asservissement sur la vitesse de déformation dans la même direction.

La vitesse de chargement et le paramètre d'amortissement sont choisis pour que les forces d'inertie restent négligeables. L'utilisation de la condition d'espace périodique (connexion numérique entre les faces opposées de l'échantillon, Cundall, 1989) permet d'éliminer les effets de bords qui peuvent être très importants. Le tableau I résume les caractéristiques initiales des échantillons dense et lâche.

Les indices des vides des échantillons dense et lâche sont proches, ce qui est dû au fait que les milieux analysés sont bidimensionnels. Cet effet a été observé par d'autres auteurs (Kruyt, 1993).

TABLEAUI États initiaux des deux échantillons dense et lâche.

Initial states of the dense and loose samples.

\begin{tabular}{c|c|c} 
Contrainte isotrope & $\begin{array}{c}\text { Échantillon } \\
\text { Dense }\end{array}$ & $\begin{array}{c}\text { Échantillon } \\
\text { Lâche }\end{array}$ \\
\hline $\begin{array}{c}\text { Coefficient du Frottement } \\
\text { intergranulaire }\end{array}$ & 0,50 & $535 \mathrm{kPa}$ \\
\hline Indice des vides initial & 0,197 & 0,50 \\
\hline Nombre de coordination & 3,774 & 3,162 \\
\hline
\end{tabular}

Il peut sembler étonnant d'obtenir un nombre de coordination inférieur à 4 aussi bien pour l'échantillon lâche que pour l'échantillon dense. En effet si l'on met en place un échantillon bidimensionnel par sédimentation chaque particule au moment de son dépôt crée 4 contacts ( 2 sur elle-même et 2 sur les particules qui la supportent). Le nombre de coordination 4 apparaît donc comme incontournable pour un milieu granulaire bidimensionnel mis en place par sédimentation. Dans le cas étudié le but recherché était de réaliser des échantillons isotropes, le mode de fabrication choisi a donc été un compactage isotrope à partir d'un état initial très dispersé. De plus, comme la pesanteur ne joue qu'un rôle négligeable dans le comportement des échantillons soumis aux contraintes de confinement utilisées, elle n'a pas été prise en compte. On sait que dans un matériau granulaire il existe un nombre non négligeable de particules non sollicitées par le réseau de forces qui équilibre les contraintes extérieures. Dans le modèle utilisé, ces particules auront un seul point de contact, parfois 2. De plus, une particule sollicitée peut être équilibrée avec seulement 2 ou 3 contacts (contacts avec frottement). Les valeurs du nombre de coordination, obtenues pour un milieu compacté de façon isotrope et non soumis à la pesanteur, seront donc en général inférieures à 4 , ce qui a été obtenu.

\section{3aits}

\section{Observations globales (à l'échelle de l'échantillon)}

Il est bien connu et admis qu'en augmentant la densitê initiale d'un matériau pulvérulent, la résistance au pic augmente mais la résistance au palier reste constante, cette dernière ne dépend pas de la densité initiale ainsi que l'indice des vides final (indice des vides critique). Il est également admis qu'en augmentant la densité initiale la tendance à la dilatation augmente.

La figure 3 montre les résultats des simulations numériques sur les échantillons dense et lâche. En concordance avec l'expérience, ces simulations montrent que la résistance au pic est une fonction de la densité initiale (Fig. 3a) et également l'indépendance de la résistance au palier par rapport à la densité initiale. Le milieu dense diminue de densité, le milieu lâche augmente de densité et les indices des vides tendent vers la même valeur à la fin de l'essai (Fig. 3c et $3 \mathrm{~d}$ ). Ces résultats sont en accord avec le concept d'indice des vides critique dans les milieux granulaires.

\section{0}

\section{Analyse des variables locales}

L'approche numérique permet d'analyser les variables locales (informations sur les contacts et les forces de contact). La figure $4 a$ montre, en particulier l'évolution du rapport des contacts glissants sur le nombre total des contacts en fonction de $\varepsilon$. Pour le matériau lâche, ce rapport reste quasiment constant tout au long de la simulation, par contre pour le milieu dense ce nombre est plus faible au début de l'essai, ce qui montre qu'un nombre significatif de glissements ne pourra se produire que lorsque la dilatance aura permis d'atteindre un état de densité suffisamment lâche.

La figure $4 \mathrm{~b}$ montre l'évolution du nombre de coordination pour les deux assemblages. Elle montre qu'un 

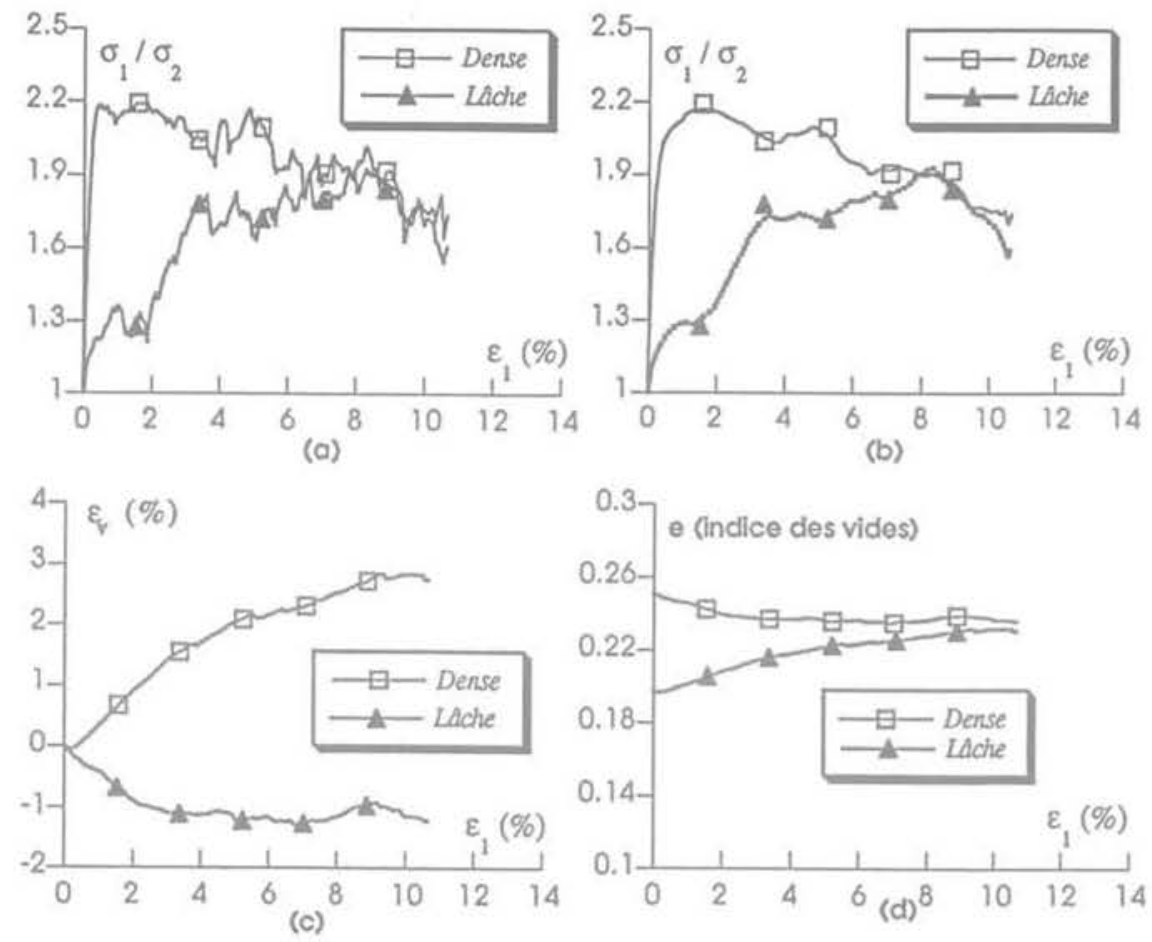

FG. 3 Comparaison des résultats des simulations sur un milieu dense et lâche, a et b) courbes brutes et lissées contrainte-déformation pour les matériaux lâche et dense, c) variation de volume pour les matériaux lâche et dense, d) variation de l'indice des vides pour les matériaux lâche et dense.

Comparison of the simulations performed on dense and loose media, a and b) rough (non smoothed) and smoothed stress-strain curves for the dense and loose materials, b) volume change curves for the dense and loose materials, d) void ratio evolution

assemblage dense perd des contacts ce qui entraîne une dilatation du milieu et qu'un assemblage lâche gagne des contacts ce qui conduit à un contractance du milieu. Un point très intéressant sur cette figure est la convergence du nombre de coordination des deux assemblages vers une valeur limite. Ce phénomène est la manifestation locale du phénomène de l'état critique dans un milieu granulaire.

\section{1 .3}

\section{Évolution de la structure interne}

Une sollicitation déviatoire engendre une anisotropie géométrique et statique dans le milieu. Ces évolutions sont accompagnées par la création de directions privilégiées, perte de contacts dans certaines directions et création dans certaines autres.

Les figures $5 \mathrm{a}, 5 \mathrm{~b}$ et $5 \mathrm{c}$ montrent, respectivement, l'évolution de la distribution du nombre des contacts pour trois étapes de l'essai biaxial. On constate qu'à l'état isotrope, la distribution du nombre des contacts est isotrope (Fig. 5a).

Après l'application de la sollicitation déviatoire, deux direction préférentielles apparaissent dans les mêmes directions que les directions principales de la sollicitation. La figure $5 \mathrm{~b}$ montre une augmentation du nombre des contacts dans la direction de la contrainte principale majeure, par contre une diminution des contacts dans la direction de la contrainte principale mineure. Globalement le nombre des contacts diminue ce qui est lié à la forte dilatance du milieu. La perte de contacts se manifeste essentiellement pour les contacts orientés dans la direction de la contrainte principale mineure,

En décharge, l'échantillon regagne une partie des contacts perdus, cependant après retour à l'état isotrope des contraintes un net déficit dans le nombre des contacts est constaté et une partie de l'anisotropie subsiste.

\section{2}

\section{Étude de l'influence du frottement local sur le comportement global}

Un milieu granulaire est un assemblage de particules avec un frottement aux contacts solides. Il est donc intéressant d'analyser l'influence de ce paramètre sur le comportement global. Dans la littérature, de nombreux débats ont eu lieu sur ce sujet.

Cinq essais ont été réalisés, en partant d'échantillons géométriquement et mécaniquement identiques, avec des coefficients de frottement intergranulaire de 0,$1 ; 0,3 ; 0,5 ; 0,7$ et 0,9 , respectivement. La figure 6 montre les résultats de ces cinq essais qui mettent en évidence une dépendance claire du comportement global du matériau vis-à-vis du frottement intergranulaire.

On constate qu'en augmentant la valeur du frottement interparticulaire $(\tan \psi)$ l'angle de frottement interne du milieu $(\phi)$ correspondant au pic augmente. Il semble que la relation entre les frottements local et global ne soit pas linéaire, ce phénomène est lié à la 


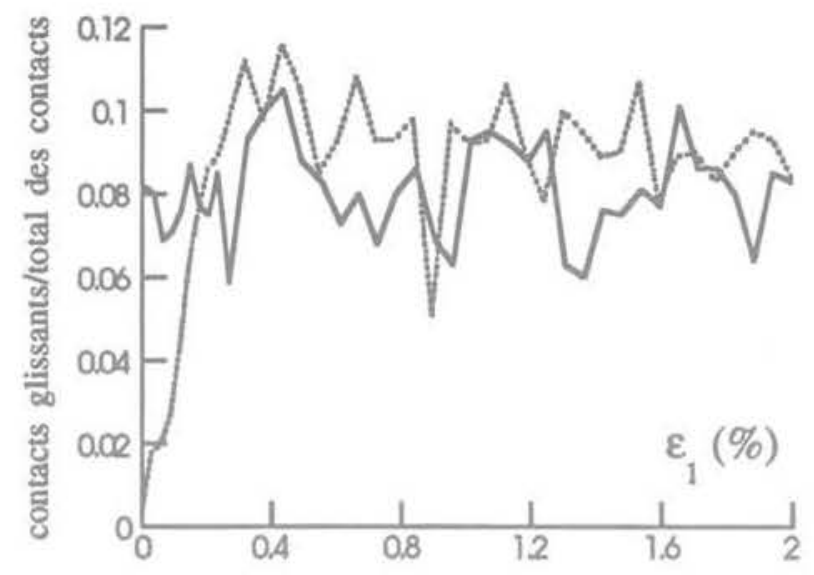

a)

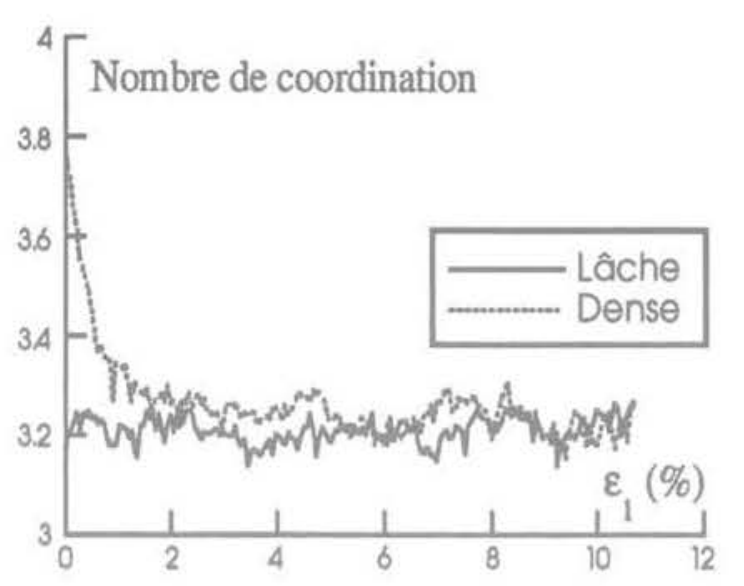

b)

FGG.4 Comparaison des informations locales obtenues lors des essais dense et lâche, a) évolution du rapport de glissement, b) évolution du nombre de coordination au cours des essais. Comparison of the local informations obtained in the dense and loose media simulations, a) evolution of the sliding ratio, b) evolution of the coordination number.

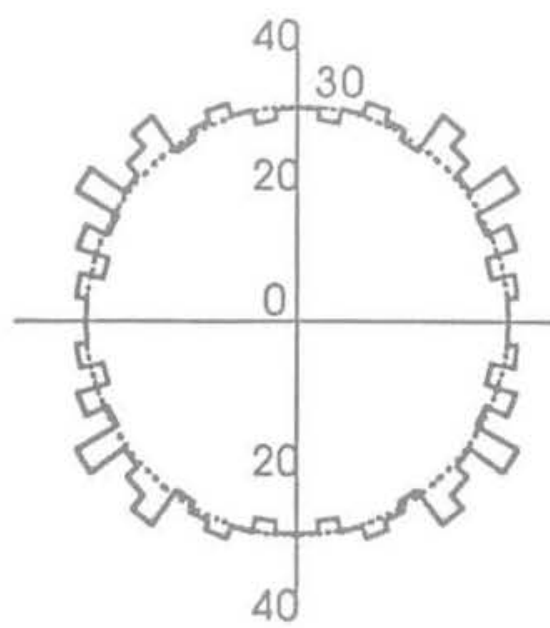

(a)

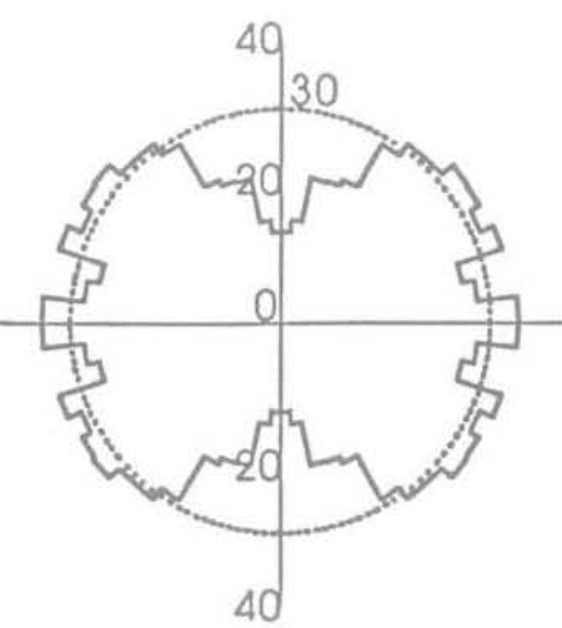

(b)

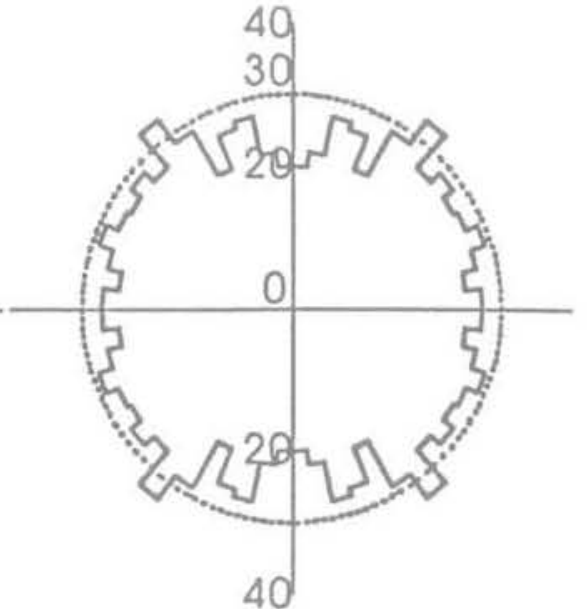

(c)

FG. 5 Évolution de Y'anisotropie géométrique, a) fin de la compression isotrope, b) après chargement déviatoire (début de décharge), c) état isotrope de contraintes après déchargement. Evolution of the geometrical anisotropy, a) at the end of the isotropic compaction, b) at the end of the deviatoric loading, c) at the end of unloading (isotropic stress state).

forte influence des rotations locales (au niveau des particules) qui sont importantes pour les matériaux avec un frottement intergranulaire élevé. Ces résultats sont en accord avec ceux obtenus par d'autres auteurs (Cundall et al., 1982; Bathurst et al., 1990; Ng et al., 1994).

Ce que nous appelons frottement interne de palier, correspond à l'angle de frottement interne à volume constant $\left(\mathrm{d} \varepsilon_{\mathrm{y}}=0\right)$ ). Les résultats (Fig. 6a) montrent qu'à l'état de déformation à volume constant, les frottements internes des différents milieux ont tendance à se rapprocher. En d'autres termes, on ne peut pas clairement faire apparaître une dépendance entre les valeurs de $\psi$ et $\phi$, il semble qu'au palier les valeurs de frottement interne soient du même ordre de grandeur. Ceci peut s'expliquer par l'anisotropie induite dans le matériau qui sera différente dans chacun des matériaux et peut contrebalancer l'effet du frottement local.

On peut aussi remarquer que l'indice des vides critique dépend du frottement intergranulaire, plus le frottement local est élevé, plus l'indice des vides critique est grand ce qui contribue à un rapprochement des frottements internes à l'état critique.

Ces résultats sont en accord avec des résultats expérimentaux d'Abriak et al. (1993) sur des rouleaux de PVC et les résultats de Skinner (1969) sur des billes de verre.

La figure 7 résume les résultats présentés dans la littérature sur l'influence du frottement intergranulaire sur le frottement interne des milieux granulaires.

L'influence du frottement local sur la dilatance a fait l'objet de travaux théoriques et expérimentaux. Les résultats obtenus sont souvent contradictoires, mais la plupart des résultats montrent une influence considérable du frottement intergranulaire sur la dilatance du milieu granulaire. La figure $6 \mathrm{~b}$ montre le comportement volumique des assemblages simulés. Elle met clairement en évidence l'influence du frottement intergranulaire sur la dilatance du matériau. Ces simulations mon- 


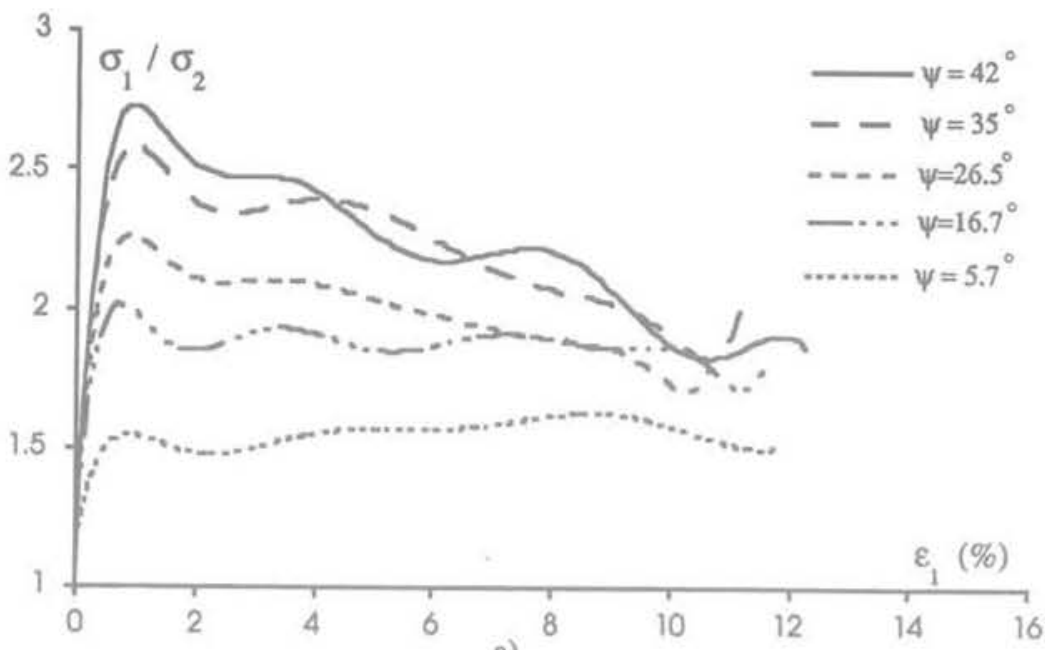

a)
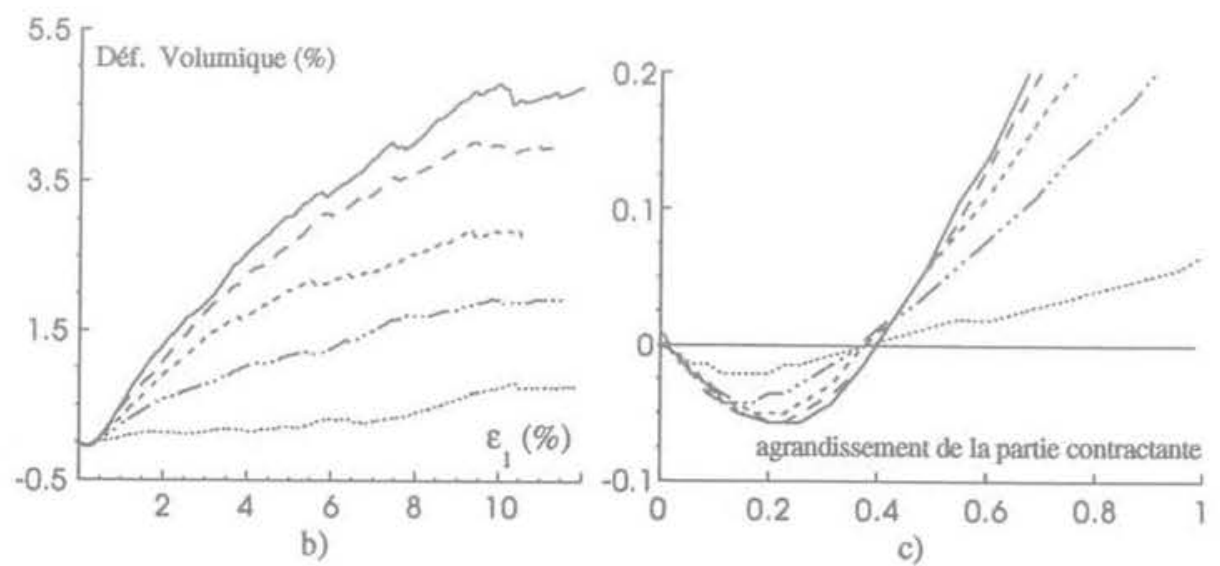

FG.6 Essai biaxiaux sur des assemblages de 1000 particules avec différents coefficients de frottement intergranulaire, a) courbes contrainte-déformation, b et c) courbes de variation de volume, Biaxial tests on a 1,000 particles packing with different intergranular friction coefficients, a) stress-strain curves, b) volume change curves.

trent qu'en augmentant le frottement local, le matériau présente une tendance plus forte à la dilatance. Ces résultats sont en bon accord avec des résultats de Bathurst et al. (1990), Kruyt (1993) et Ng et al. (1994).

Une étude complète de l'influence du blocage des rotations a été menée (Mahboubi, 1995). Ce blocage entraîne une forte augmentation de la résistance au pic et au palier, l'augmentation de la zone de contractance initiale ainsi que de la pente de dilatance du milieu.

\section{4}

\section{Simulation d'essais triaxiaux (milieux tridimensionnels)}

L'assemblage de base est constitué de 1000 particules sphériques qui sont en interaction aux points de contacts. La modélisation du contact se fait par la loi de Hertz avec frottement Coulombien. La distribution des particules et les tailles et caractéristiques des particules sont celles des essais bidimensionnels. Après la génération aléatoire des particules dans un cube, les étapes suivantes sont suivies pour obtenir des échantillons ayant les indices des vides désirés.

i) compactage de l'échantillon en diminuant le volume de l'espace périodique de façon isotrope avec un coefficient de frottement intergranulaire quasiment nul pour faciliter le compactage du milieu;

ii) pour obtenir un échantillon dense, le compactage est poursuivi avec le même coefficient de frottement intergranulaire quasi nul jusqu'à l'obtention d'un indice des vides égal à 0,64 et d'une contrainte isotrope égale à $76 \mathrm{kPa}$. Ensuite le coefficient de frottement intergranulaire est pris égal à 0,5 .

iii) pour obtenir un échantillon lâche, le milieu obtenu en (i) est compacté en considérant un coefficient de frottement égal à 0,5 jusqu'à l'obtention d'un indice des vides égal à 0,73 et une contrainte isotrope égale à $70 \mathrm{kPa}$.

Le tableau II résume les états initiaux des deux échantillons, dense et lâche.

\begin{tabular}{|c|c|c|c|c|}
\hline \multirow[t]{2}{*}{ TABLEAU II } & \multicolumn{4}{|c|}{$\begin{array}{l}\text { États initiaux des deux échantillons dense } \\
\text { et lâche. } \\
\text { Initial states of the dense and loose sample }\end{array}$} \\
\hline & $\begin{array}{l}\text { Contrainte } \\
\text { Isotrope }\end{array}$ & $\tan (\psi)$ & $e_{0}$ & $\begin{array}{c}c \\
\text { (nombre de) } \\
\text { coordination) }\end{array}$ \\
\hline $\begin{array}{l}\text { Échantillon } \\
\text { Dense }\end{array}$ & $76(\mathrm{kPa})$ & 0,5 & 0,64 & 5,05 \\
\hline $\begin{array}{l}\text { Échantillon } \\
\text { Lâche }\end{array}$ & $70(\mathrm{kPa})$ & 0,5 & 0,73 & 4,25 \\
\hline
\end{tabular}




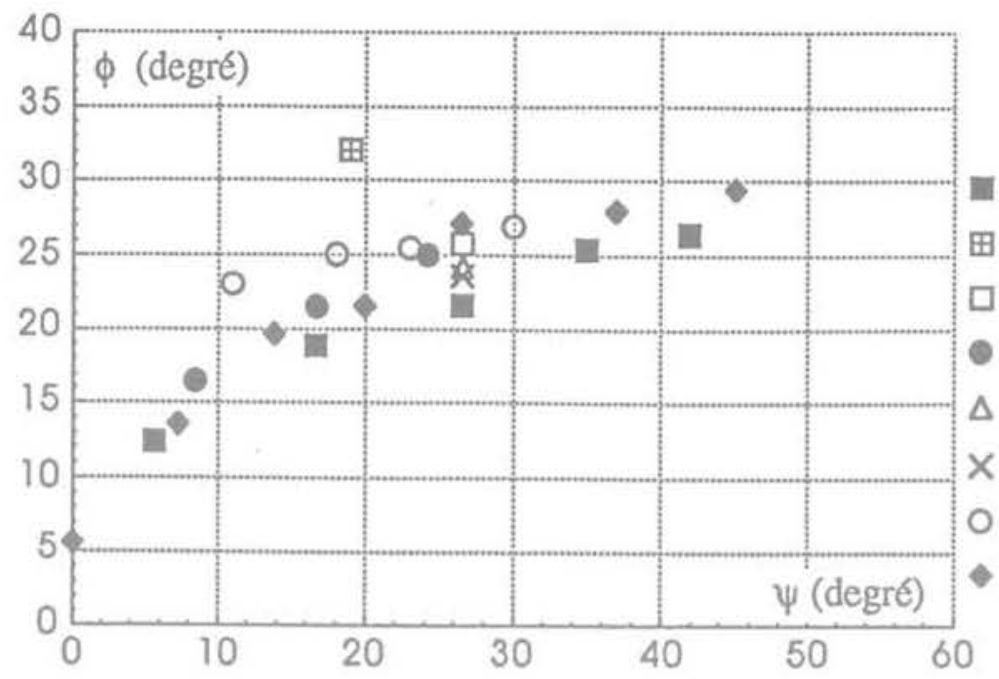

Angle de frottement interne au pic

Nos simulations (biax.)

Matsuoka (Exp., biax)

Rothenburg (sim., biax.)

Knuyl (sim, biax)

Cundall (sim., Cis.)

Cundall (sim., biax.)

Abriak (Exp., biax.)

Yemmas (sim., biax.)

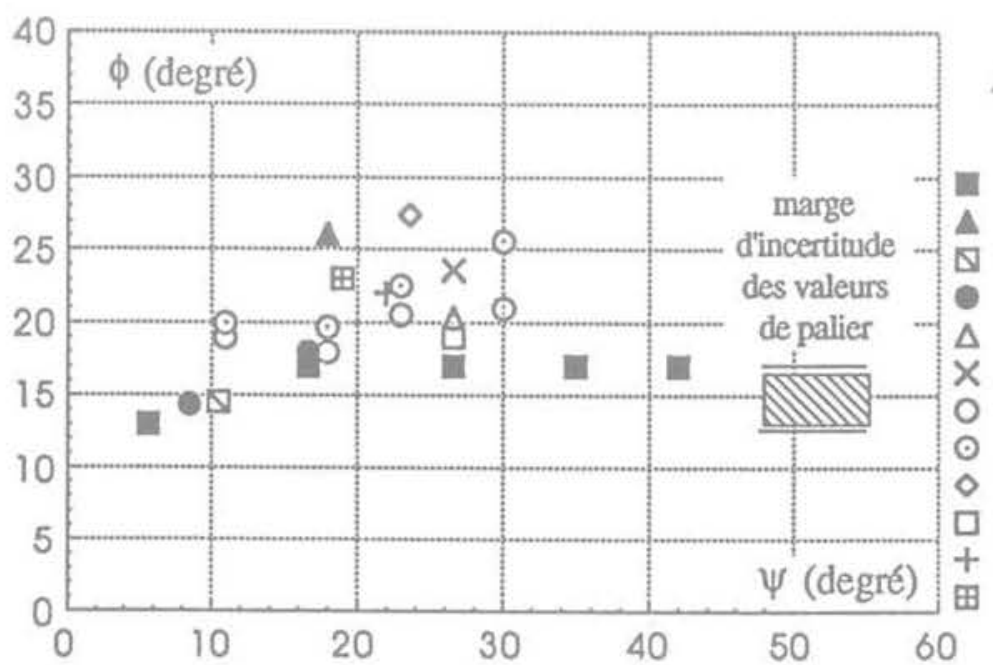

Angle de frottement

interne au palier

Nos simulations (biax.)

Gourves (Exp., biax.)

chapuis (Exp., biax.)

Kruyt (sim, biax.)

Cundall (sim., cis.)

Cundall (sim., biax)

Abriak (Exp., cis.)

Abriak (Exp., biax.)

Thornton(sim., biax.)

Rothenburg (sim., biax)

oda (Exp.-cis.)

Matsuoka (Exp.-cis.)

Résumé des travaux sur l'influence du frottement local sur le frottement global correspondant au pic et au palier (Exp. = Expérience, Sim. = Simulation, Biax. = Biaxial, Cis. = Cisaillement $)$.

Summary of the different results on the influence of the intergranular friction on the internal friction for the peack and for the residual state (Exp. = Experience, Sim. = Simulation, Biax. $=$ Biaxial test, Cis. $=$ Shear test .

\section{1}

\section{Simulation d'essais de compression axisymétrique sur des échantillons dense et lâche}

\section{1 .1}

\section{Observations globales}

La convergence des indices des vides des deux assemblages vers une valeur unique est clairement mise en évidence dans ces simulations (Fig. 8). Expérimentalement, il est souvent difficile de mettre en évidence l'état critique car l'apparition de bandes de cisaillement (localisation des déformations) perturbe I'homogénéité des champs de contraintes et de déformations dans l'échantillon (Desrues, 1993). Le mode d'application du chargement (champ linéaire de dépla- cement des particules) et les bornes périodiques empêchent la formation de bandes de cisaillement dans la simulation numérique (Bardet et al., 1991).

La pente initiale des courbes de chargement est très raide. On constate d'autre part que l'évolution du volume de l'assemblage dense ne présente presque plus de zone de contractance et que le milieu devient rapidement dilatant. Ceci peut être expliqué par la structure particulière de l'assemblage dense avec un nombre réduit de particules qui ne permet pas de faire apparaître des mécanismes entraînant la contractance du milieu. Ce phénomène a été noté par d'autres chercheurs (Chen et al., 1993).

\section{1 .2}

\section{Observations locales}

Au niveau local la sollicitation déviatoire est accompagnée par les phénomènes suivants: 

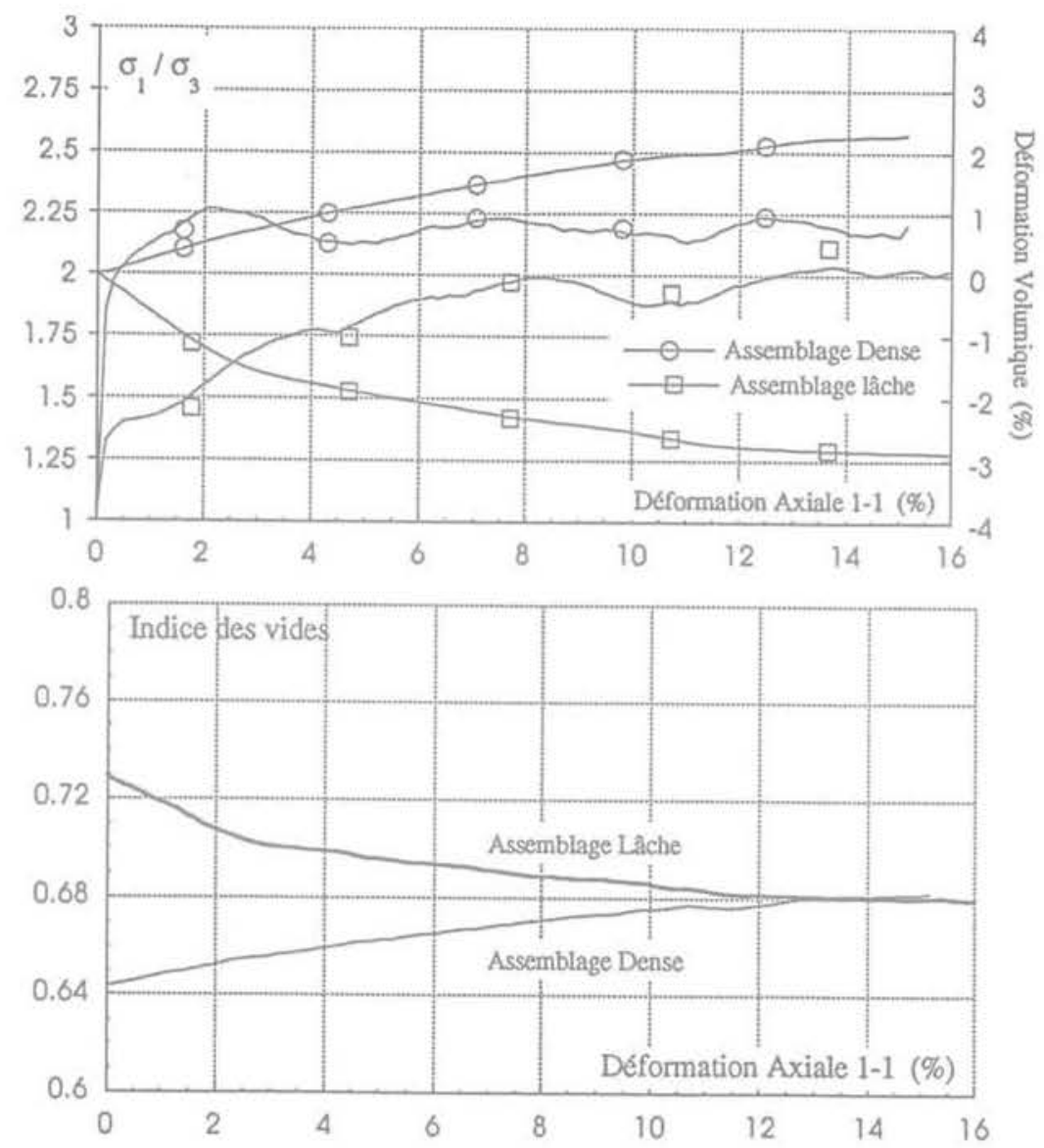

FG.8. Simulation d'essais triaxiaux axisymétriques sur échantillons lâche et dense.

Simulation of axisymetric triaxial tests on loose and dense samples.

- la concentration des contacts et l'augmentation des forces de contact dans la direction de la contrainte majeure;

- la perte de contact (Fig. 9a) et l'augmentation des contacts glissants (Fig. 9b) dans la direction de la contrainte mineure.

On peut constater que la stabilisation de la courbe de chargement est accompagnée par la stabilisation du nombre de coordination (manifestation locale de l'état critique) et du nombre de contacts glissants.

L'ensemble de ces phénomènes s'accompagne du développement d'une anisotropie (géométrique et statique) dans le milieu granulaire.

\section{2}

\section{Simulation d'essais véritablement triaxiaux}

L'une des utilisations très intéressantes de la simulation numérique discrète des milieux granulaires consiste à utiliser cet outil comme un appareil véritablement triaxial pour réaliser des chemins de sollicitation qui ne sont pas faciles à réaliser expérimentalement.
Les études du comportement des matériaux granulaires par un appareil véritablement triaxial sont rares. Lanier a réalisé de tels essais (Cambou et Lanier, 1988), Les résultats mettent en évidence l'influence de l'anisotropie induite sur le comportement des matériaux granulaires. Nous présentons les résultats de différents chemins de sollicitation sur un assemblage dense de 1000 particules caractérisé par le tableau II et dont l'état initial est dense. Les chemins de sollicitation sont définis par le coefficient de contrainte déviatoire, b, défini par:

$$
\mathrm{b}=\frac{\mathrm{s}_{22}-\mathrm{S}_{33}}{\mathrm{~s}_{11}-\mathrm{s}_{33}}
$$

avec:

$$
\mathrm{s}_{\mathrm{i}}=\sigma_{\mathrm{t}}-\frac{1}{3} \sigma_{\mathrm{k} 2} \delta_{i j} \text { et } \mathrm{s}_{11} \geq \mathrm{s}_{22} \geq \mathrm{s}_{30}
$$

Ainsi $b=0$ correspond à un essai de compression dans la direction 1 et $\mathrm{b}=1$ à une sollicitation d'extension dans la direction 3 (Fig. 14). Il existe six chemins de sollicitations correspondant à chaque valeur de b. Par raison de symétrie, il existe seulement trois chemins de sollicitations correspondant aux valeurs de $b=$ 0 et $b=1$. 

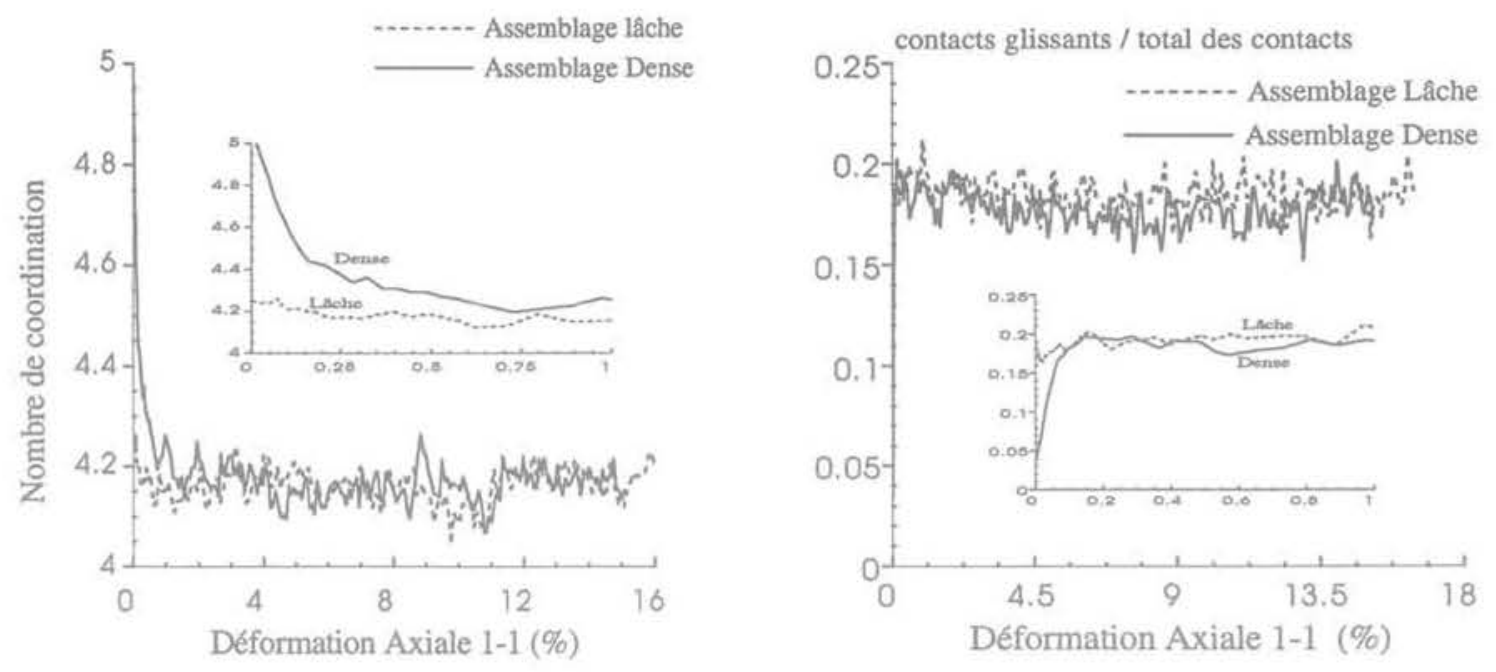

FG.9 Evolution du nombre de coordination et du rapport des contacts glissants au cours de l'essai pour les assemblages dense et lâche.

a) evolution of the coordination number for the dense and loose samples, b) evolution of the sliding ratio of the dense and loose samples.

\section{Recherche du critère d'écoulement (plasticité parfaite)}

Le critère d'écoulement est l'enveloppe de toutes les surfaces de charge possibles. On peut considérer qu'il correspond à un état dans lequel la variable interne (variable d'écrouissage) a atteint sa limite licite. L'assemblage granulaire est sollicité suivant différents chemins de sollicitations, jusqu'à atteindre l'écoulement libre correspondant à une stabilisation de la courbe effort-déformation. Cinq chemins différents ont été explorés pour obtenir cinq points du critère. La figure (10) montre, point par point, les différents chemins de sollicitation. Les chemins effectués correspondent à $\mathrm{b}=0 ; 0,125 ; 0,25 ; 0,5$ et 1 .

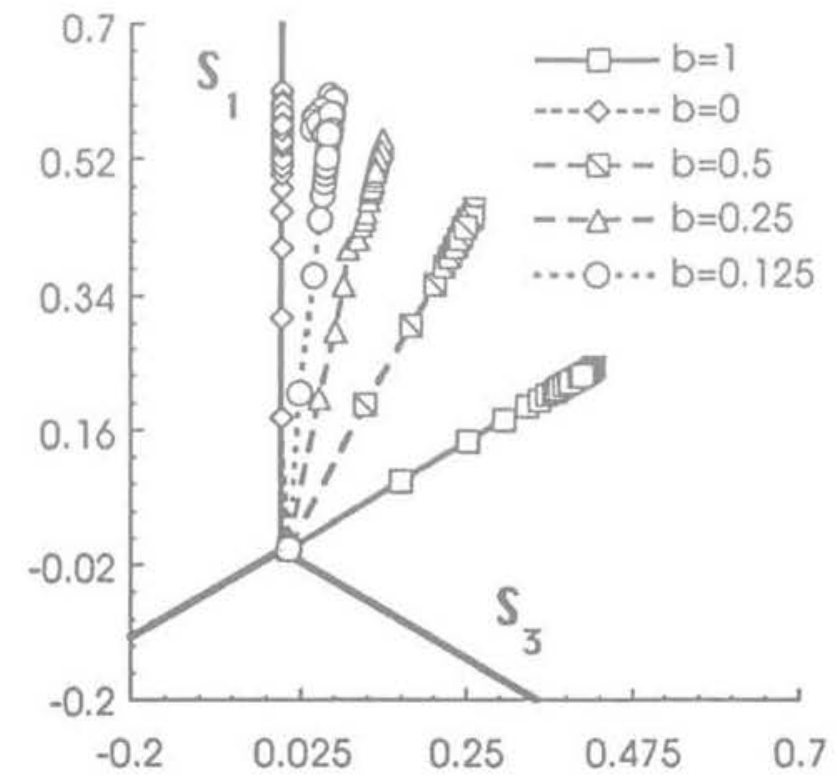

FiG.10 Différents chemins de sollicitations effectués.

Representation of the different loading paths in the deviatoric plane.
Les figures (11a) et (11b) montrent les courbes effort-déformation et l'évolution des déformations volumiques de l'assemblage au cours de ces sollicitations. On peut constater que les courbes de variation de volume (Fig. 11b) sont quasiment confondues et donc on peut supposer que pour un matériau initialement isotrope le rapport $\frac{\mathrm{d} \varepsilon_{\mathrm{v}}}{\mathrm{d} \varepsilon_{11}}$ reste constant et ceci quelque soit le chemin de sollicitation radial. Ce résultat est en bon accord avec les résultats expérimentaux de Lanier (1988). Dans ces figures, $e_{11}$ et $S_{\| 1}$ sont définis par:

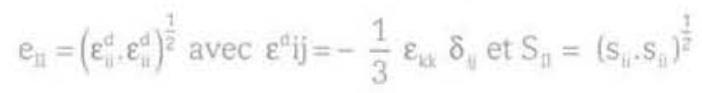

La figure 12 montre les points extrêmes de chaque chemin de sollicitation correspondant à la limite d'écoulement. La comparaison avec les différents critères souvent utilisés dans le domaine de la mécanique des sols montre une bonne concordance avec le critère de Lade (Lade et al., 1975) ou le critère du modèle CJS (Cambou et Jafari, 1988).

Le critère de Mohr-Coulomb apparait comme une approximation acceptable mais sous-estimant de façon sensible la limite de rupture pour des chemins de sollicitations dont la valeur de b est comprise entre 0,25 et 0,75 .

\subsection{2}

\section{Anisotropie induite et évolution des surfaces de charge}

Les résultats expérimentaux de Lanier (1988) mettent clairement en évidence l'influence de l'anisotropie induite sur le comportement d'un milieu granulaire. Pour tester la capacité de la MED à mettre en évidence le comportement des matériaux granulaires sur des chemins de sollicitation complexes, des essais similaires aux essais de Lanier ont été simulés par la MED.

La première étape consiste à préparer un échantillon ayant une anisotropie induite qui constituera l'état initial pour différents chemins de sollicitations. Pour cela, l'échantillon dense de 1000 particules est soumis à 


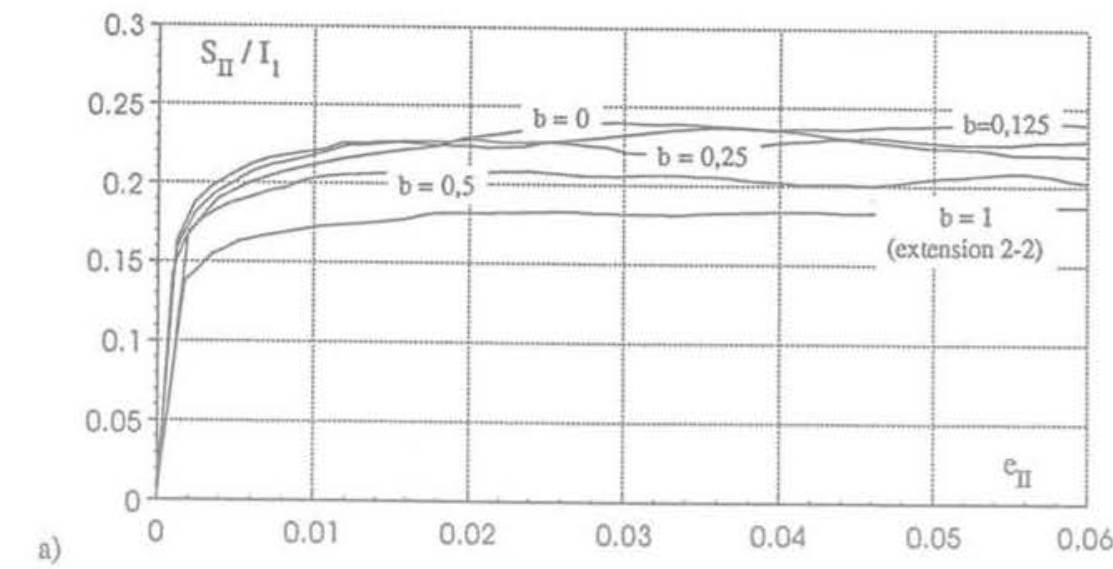

a)

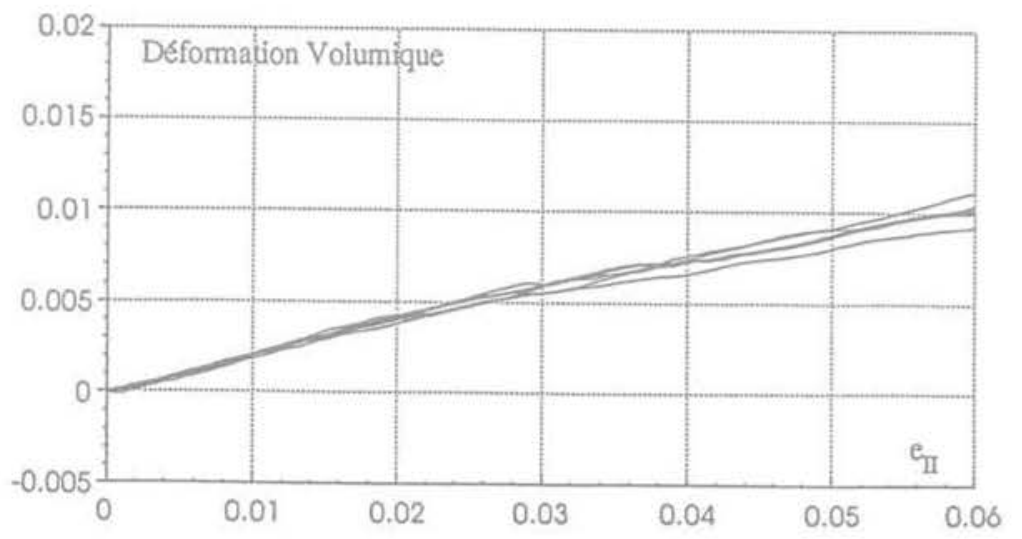

FIG. 11 Courbes contrainte-déformation et évolution du volume pour différents chemins de sollicitations effectués.

Global responses of the different loading paths.

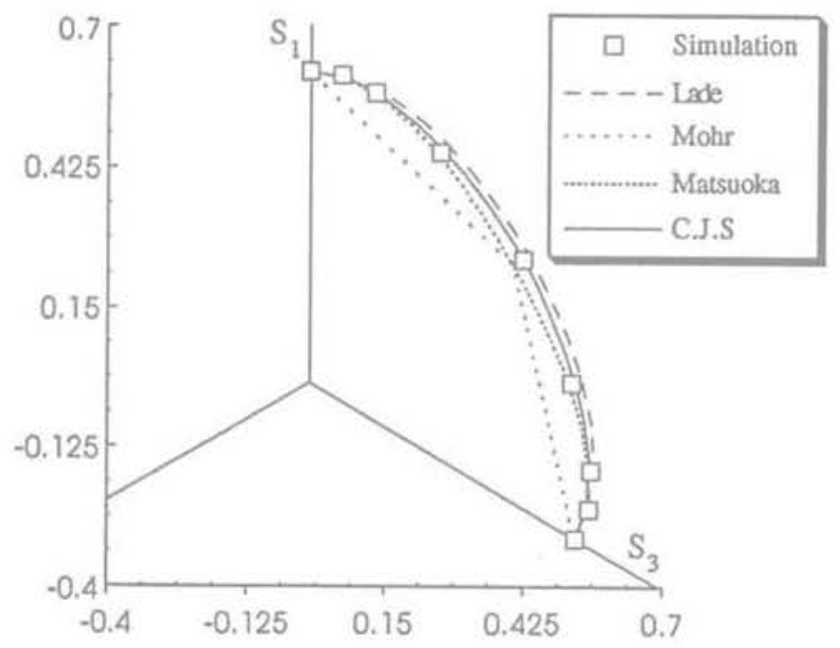

FG. 12 Comparaison entre le critère obtenu et quelques autres critères. Comparison between the obtained criterion and classical criteria.

une sollicitation de compression axisymétrique dans la direction 1 jusqu'à une déformation axiale égale à $\varepsilon_{1}$ $=0,10$ (point $\mathrm{A}$ sur la figure 13), l'échantillon est ensuite déchargé dans la même direction pour atteindre l'état de contrainte isotrope initial (point B sur la figure 13). La déformation axiale est suffisamment grande pour engendrer une anisotropie induite importante dans
I'assemblage. A cette étape (point B sur la figure 13) l'échantillon est soumis à différents chemins de chargement (définis par différentes valeurs de b) de façon à analyser le comportement du matériau sous l'influence de l'anisotropie induite précédemment (Fig. 14).

Nous comparons sur les figures 15,16 et 17 des essais réalisés pour des chemins de sollicitations ayant la même valeur de b. Les différentes courbes tracées sur chaque figure seraient nécessairement identiques (aux erreurs et incertitudes expérimentales près) pour un milieu initialement isotrope. L'ensemble des courbes montre l'effet très important de l'anisotropie induite par la sollicitation $\mathrm{S}_{0}$. Globalement l'ensemble des résultats montre que :

- les pentes initiales des courbes $S_{\mu} / I_{1}=f\left(e_{n}\right)$ diminuent quand le chemin de sollicitation s'ecarte du chemin initial qui a induit l'anisotropie;

- l'ensemble des courbes $S_{n 1} / I_{1}=f\left(e_{n}\right)$ convergent vers une valeur d'écoulement limite unique ce qui semble indiquer que au cours de la sollicitation l'anisotropie initiale évolue et que lorsqu'on atteint la limite de l'écoulement libre l'état interne limite parait indépendant de l'état interne initial;

- l'ensemble des courbes caractérisant les variations de volume fait apparaitre un accroissement très important de la zone de contractance quand le chemin de sollicitation s'écarte du chemin initial qui a induit l'anisotropie;

- les pentes de dilatance obtenue après le domaine de contractance semblent indépendantes de l'anisotropie initiale et du chemin de sollicitation suivi. 


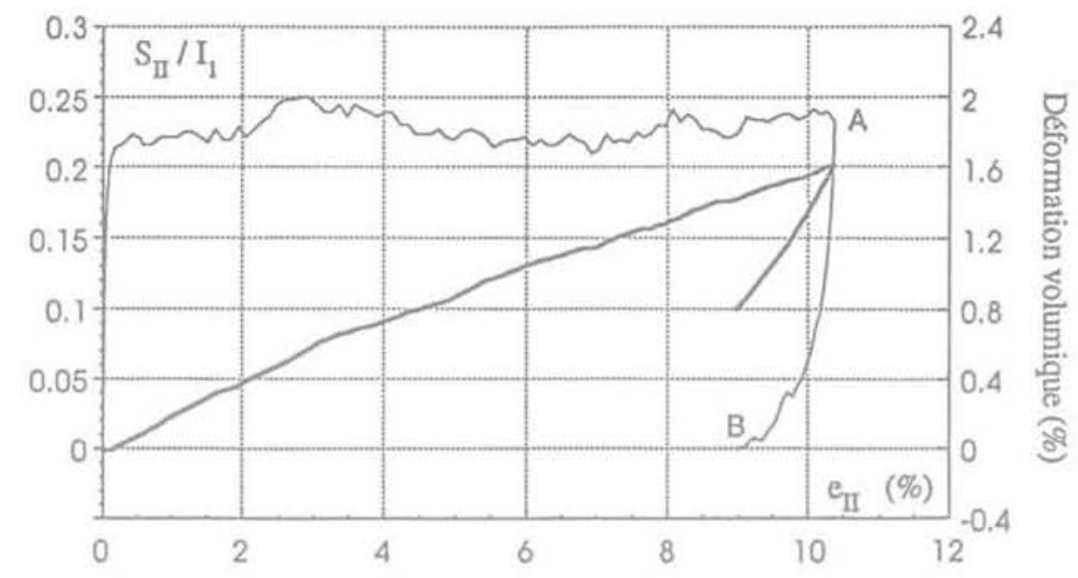

FG. 13 Sollicitation initiale dans la direction $1: S_{0}$. Initial loading in the direction $1\left(\mathrm{~S}_{0}\right)$.

Une représentation condensée des résultats de l'ensemble des simulations est présentée sur la figure 18. Sur cette figure les lignes isovaleurs de $e_{11}\left(e_{1 I}\right.$ $=1 ; 2 ; 4$ et $8 \%$ ) ont été tracées pour différents chemins de chargement. Cette représentation permet de caractériser l'allure de l'évolution des surfaces de charge dans un matériau granulaire. Ces surfaces sont enveloppées par la surface d'écoulement limite (critère de rupture). La forme des surfaces ainsi définies semble être homothétique de la surface limite d'écoulement. Les centres de gravité de ces surfaces sont localisés dans la direction de l'anisotropie induite par le premier chargement. L'évolution de ces surfaces est similaire à celle obtenue par un écrouissage cinématique dans une modélisation élasto-plastique.

Les résultats obtenus sont qualitativement comparables avec ceux obtenus expérimentalement par Lanier (1988),

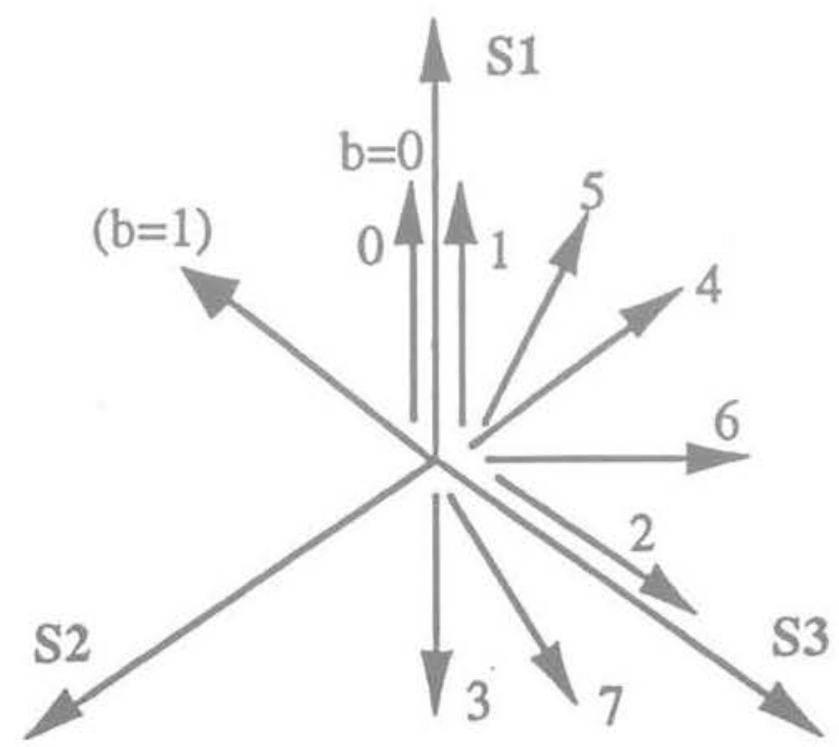

FIG,14 Représentation graphique des chemins de sollicitations effectués dans le plan déviatoire.

Representation of the different loading paths in the deviatoric plane.

\section{5}

\section{Simulation d'essais non homogènes (écoulement dans un silo)}

La méthode des éléments discrets présente un grand intérêt dans l'analyse de problèmes aux limites dans lesquels le gradient des variables continues $(\sigma, \varepsilon)$ est non négligeable pour une dimension voisine de la taille des particules. Dans ce cas les phénomènes locaux discrets sont prépondérants et seule une analyse prenant en compte le milieu discret sera capable de mettre en lumière certains phénomènes apparaissant à l'échelle globale. Nous présentons un exemple d'application dans le domaine du stockage des matériaux granulaires dans un silo.

L'écoulement gravitaire d'un milieu granulaire dans un silo a été modélisé à l'aide de la méthode des éléments discrets. La configuration utilisée est montrée sur la figure 19, elle servira à étudier l'effet de l'humidité dans le milieu sur la cinématique de l'écoulement lors de la décharge. De même, l'influence de la largeur d'ouverture sur les possibilités d'écoulement est analysés. Le modèle proposé est bidimensionnel et symétrique, L'introduction de l'humidité dans le milieu a nécessité l'intégration dans la méthode des éléments discrets du calcul des forces capillaires.

Les parois du fond du silo sont inclinées par rapport à l'horizontale de $15^{\circ}$ (Fig. 19) et trois largeurs d'ouverture différentes sont utilisées. Ces largeurs sont caractérisées par le paramètre $\mathrm{b} / \mathrm{D}_{0}$ défini comme le rapport entre la largeur d'ouverture et le diamètre moyen de particules $\left(\mathrm{b} / \mathrm{D}_{0}=4 ; 6,5\right.$ et 8,33$)$. Les études ont été menées sur le milieu sec $\left(w_{j}=0\right)$ et sur le milieu humide avec une teneur en eau $\mathrm{w}_{\mathrm{b}}=0,30 \%$. Pour une ouverture étroite $\left(\mathrm{b} / \mathrm{D}_{\mathrm{b}}=4\right)$ l'écoulement ne se produit ni pour le matériau humide, ni pour le matériau sec. Une arche stable se forme au niveau de l'ouverture qui empêche l'écoulement du matériau ensilé (Fig. 20a, 20b).

Pour une ouverture plus large $\left(\mathrm{b} / \mathrm{D}_{0}=8,33\right)$ le silo se vide, que le matériau soit sec ou humide. Au cours de l'écoulement, une cuvette en forme d'entonnoir se développe au centre du silo (Fig. 21). Les grains situés au centre du silo sont entraînés par l'écoulement alors qu'une zone située près des parois n'est pas mobili- 

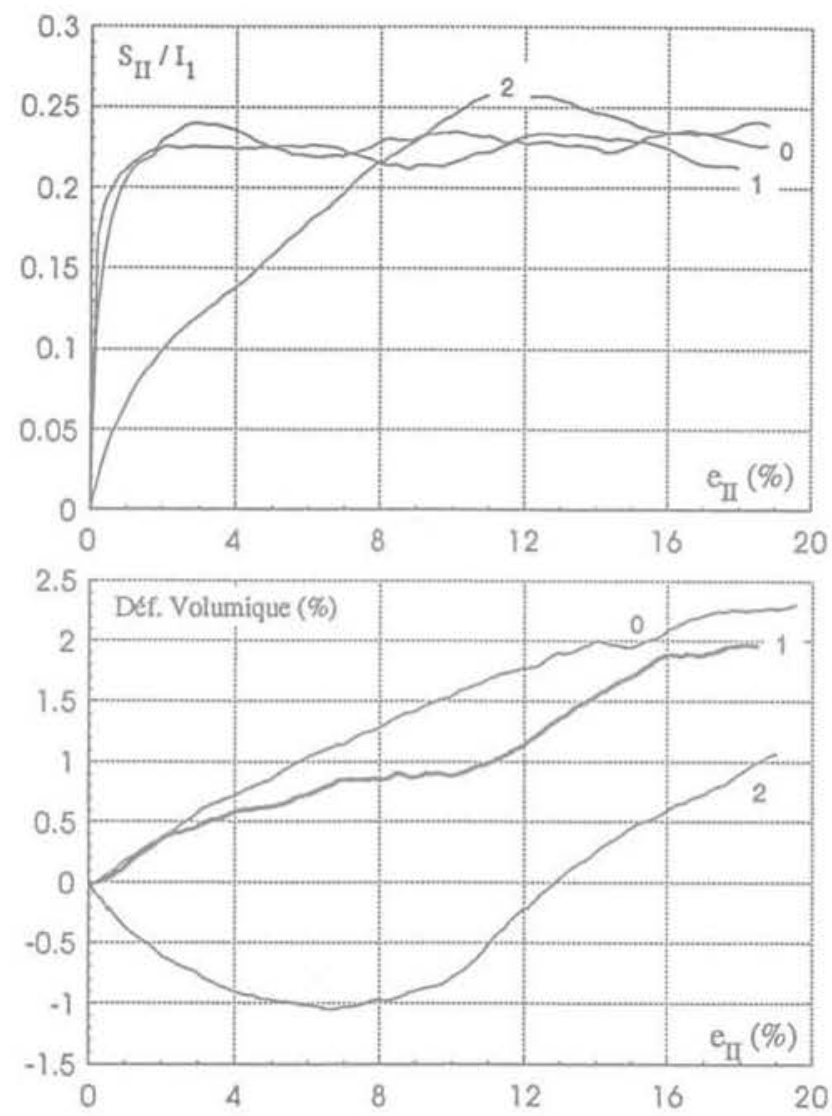

FIG. 10 Courbes de chargement et variation du volume pour trois essais d'extension axisymétriques $(b=1)$ :

Courbes 0 : chemin $n^{\circ} 3$ (état vierge).

Courbes 3 : chemin $n^{\circ} 3$ précédé par le chemin $\mathrm{n}^{\circ} 0$.

Courbes 4 : chemin $n^{\circ} 4$ précédé par le che$\min n^{\circ} 0$.

(Les numéros des chemins sont définis sur la figure 14.)

Resistance and volume change curves of three axisymetric extension tests $(\mathrm{b}=1)$ :

Curve 0 : path $n^{\circ} 3$, virgin state.

Curve 3 ; path $n^{\circ} 3$ with previous loading on path

Curve 4 ; path $n^{\circ} 4$ with previous loading on path 0 .

(The numbers of the paths are indicated on figure 14.).
FG.15 Courbes de chargement et variation du volume pour trois essais de compression axisymétriques $(\mathrm{b}=0)$ :

Courbes 0 : compression axisymétrique dans la direction 1 (état vierge) chemin $n^{\circ} 0$. Courbes 1 : chemin $n^{\circ} 1$ précédé par le che$\min \mathrm{n}^{\circ} 0$.

Courbes 2 : chemin $n^{\circ} 2$ précédé par le che$\min n^{\circ} 0$.

(Les numéros des chemins sont définis sur la figure 14.)

Resistance and volume change curves of three axisymetric compression tests $(\mathrm{b}=0)$ :

Curve 0 : axisymetric compression test in direc tion 1 (path $n^{\circ} 0$, virgin state).

Curve 1 : path $n^{\circ} 1$ with previous loading on path 0.

Curve 2: path $n^{\circ} 2$ with previous loading on path 0

(The numbers of the paths are indicated on figure 14.)
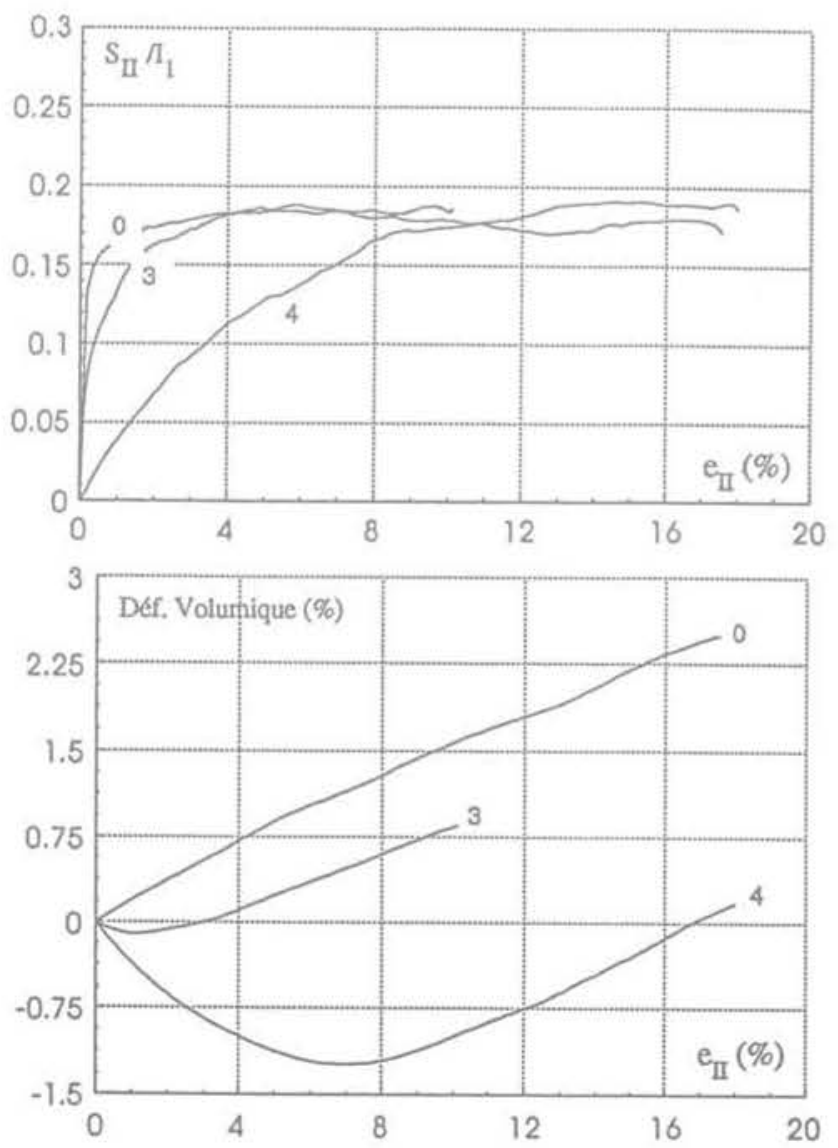

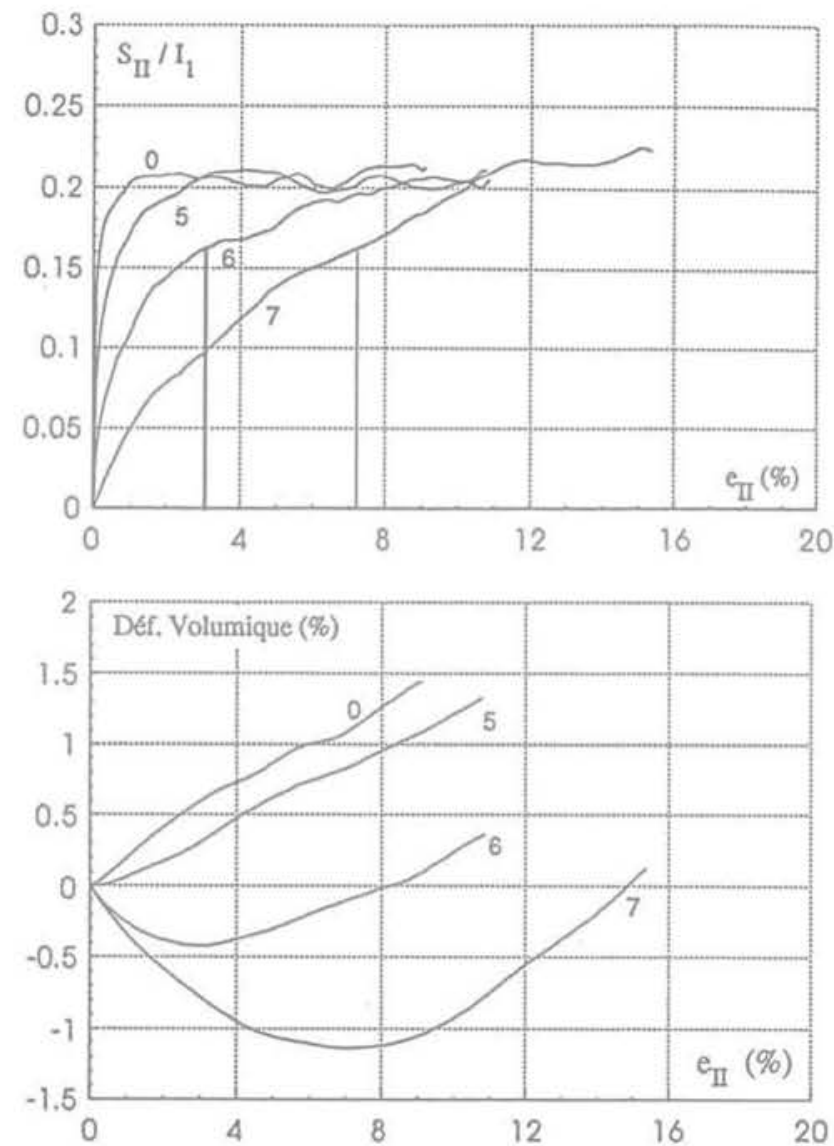

FG. 17 Courbes de chargement et variation du volume pour trois simulations avec $\mathrm{b}=$ 0,50 :

Courbes 0 : chemin $n^{\circ} 5$ (milieu vierge). Courbes 5 : chemin $n^{\circ} 5$ précédé par le che$\min n^{\circ} 0$.

Courbes 6 : chemin $n^{\circ} 6$ précédé par le che$\min \mathrm{n}^{\circ} \mathrm{O}$

Courbes 7 : chemin $n^{\circ} 7$ précédé par le che$\min \mathrm{n}^{\circ} 0$.

(Les numéros des chemins sont définis sur la figure 14.)

Resistance and volume change curves of three tests identified by $b=0.50$ :

Curve 0 : path $n^{\circ} 5$, virgin state.

Curve 5 : path $n^{6} 5$ with previous loading on path 0.

Curve 6 : path $n^{\circ} 6$ with previous loading on path 0 .

Curve 7 : path $n^{\circ} 7$ with previous loading on path 0.

(The numbers of the paths are indicated on figure 14.)

sée. L'angle qui délimite ces deux zones évolue au cours de la décharge. Dans le cas d'un matériau humide la zone en écoulement est plus étroite.

A la fin de la décharge, des grains restent en équilibre sur le fond du silo car l'angle de frottement interne du milieu qui peut être approché par l'angle de talus naturel $\left(\phi \approx 30^{\circ}\right)$ est supérieur à l'inclinaison du fond du silo (Fig. 22). Dans le cas du matériau humide le volume de matériau non vidangé est plus important.

Pour une ouverture intermédiaire $\left(\mathrm{b} / \mathrm{D}_{0}=6,5\right)$ l'écoulement se produit dans le cas du matériau sec tandis que pour le matériau humide une arche se

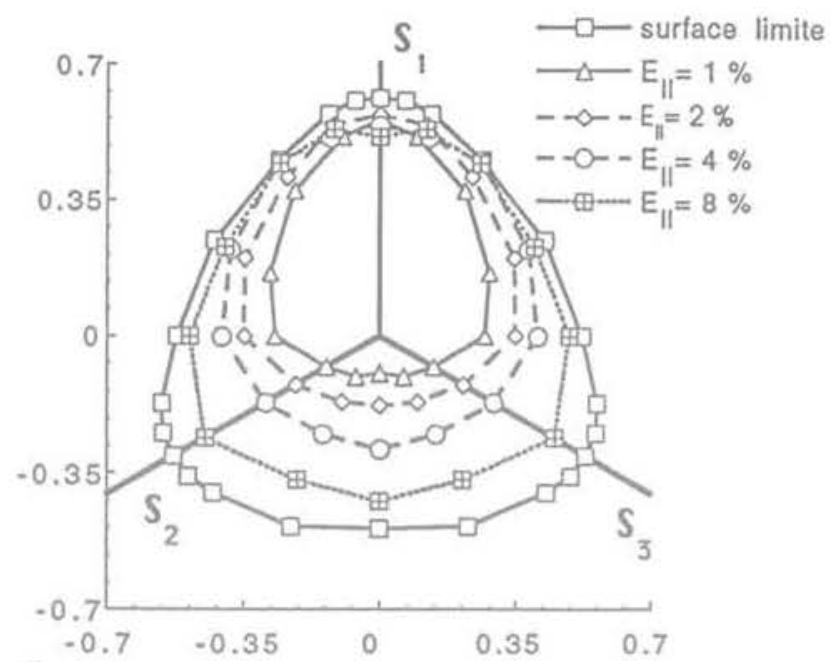

a)

b)

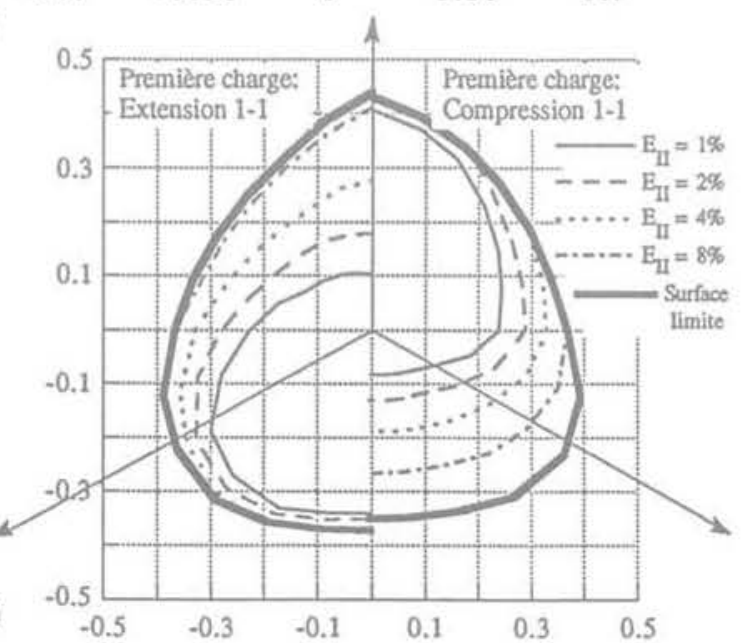

FIG. 18 Courbes isovaleurs pour différentes valeurs de la déformation, a) résultats numériques, b) résultats expérimentaux de Lanier (1989).

Isovalue curves for different strains, al numerical simulations, b) experimental results obtained by Lanier (1988).

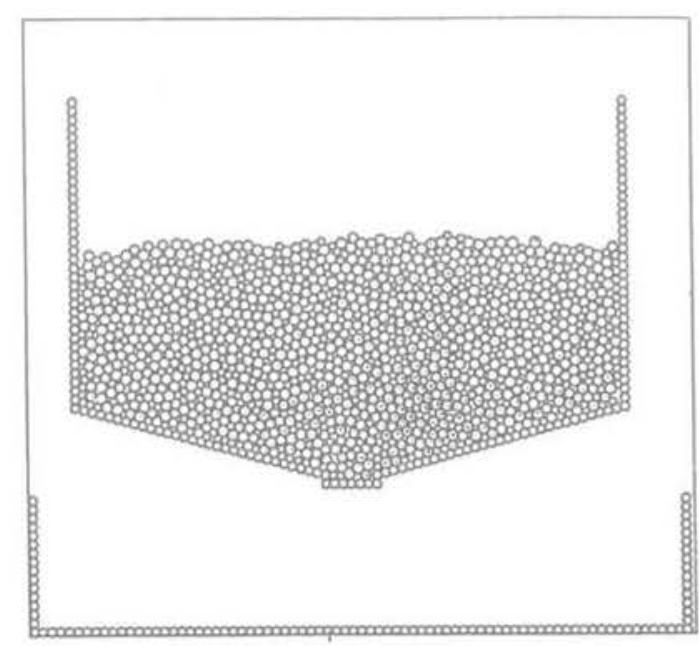

FiQ. 19 Configuration initiale du silo et de l'assemblage granulaire. Initial configuration of silo and granular packing 

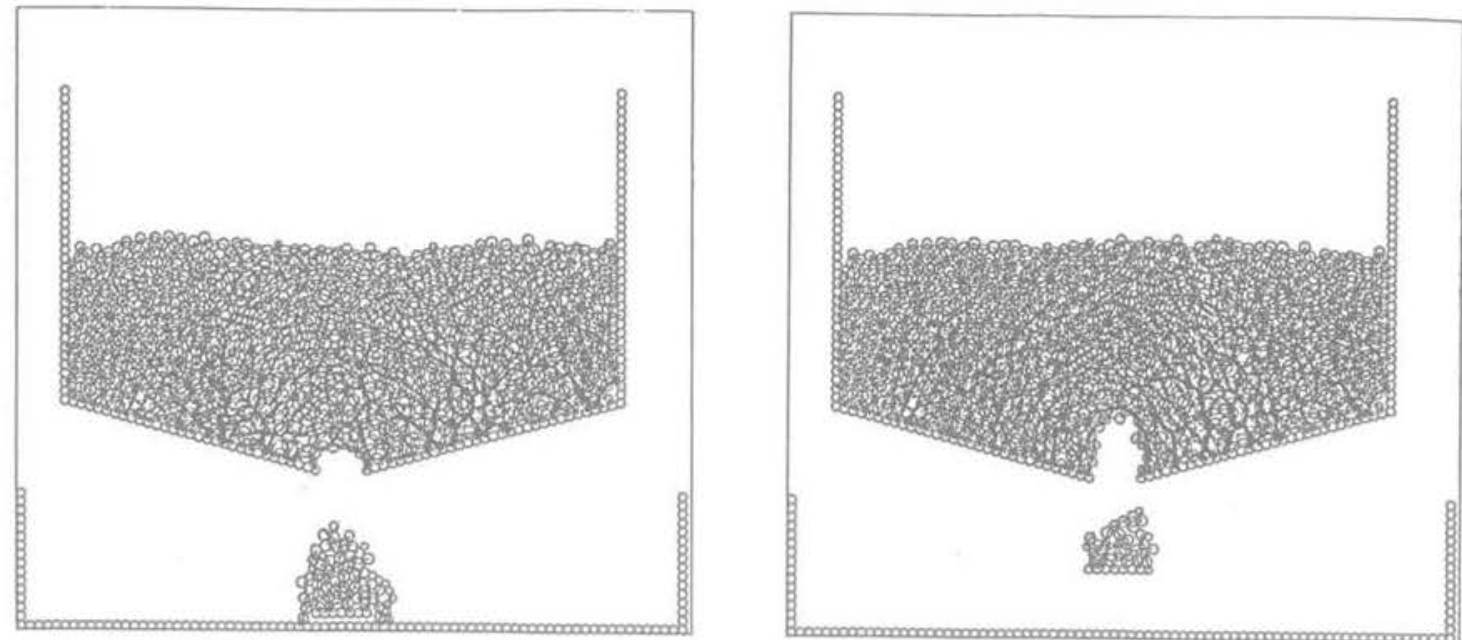

FiG. 20 Écoulement bloqué à travers une ouverture étroite $\left(\mathrm{b} / \mathrm{D}_{0}=4\right)$, a) matériau $\left.\mathrm{sec}, \mathrm{b}\right)$ matériau humide (les traits noirs sur les particules sont proportionnels aux forces de contact).

Blocking of the granular flow throughout a narrow openning $\left(\mathrm{b} / \mathrm{D}_{\mathrm{f}}=4\right)$, a) dry materials, b) wet materials (the black strokes between the centers of the particles are proportional to the contact forces).
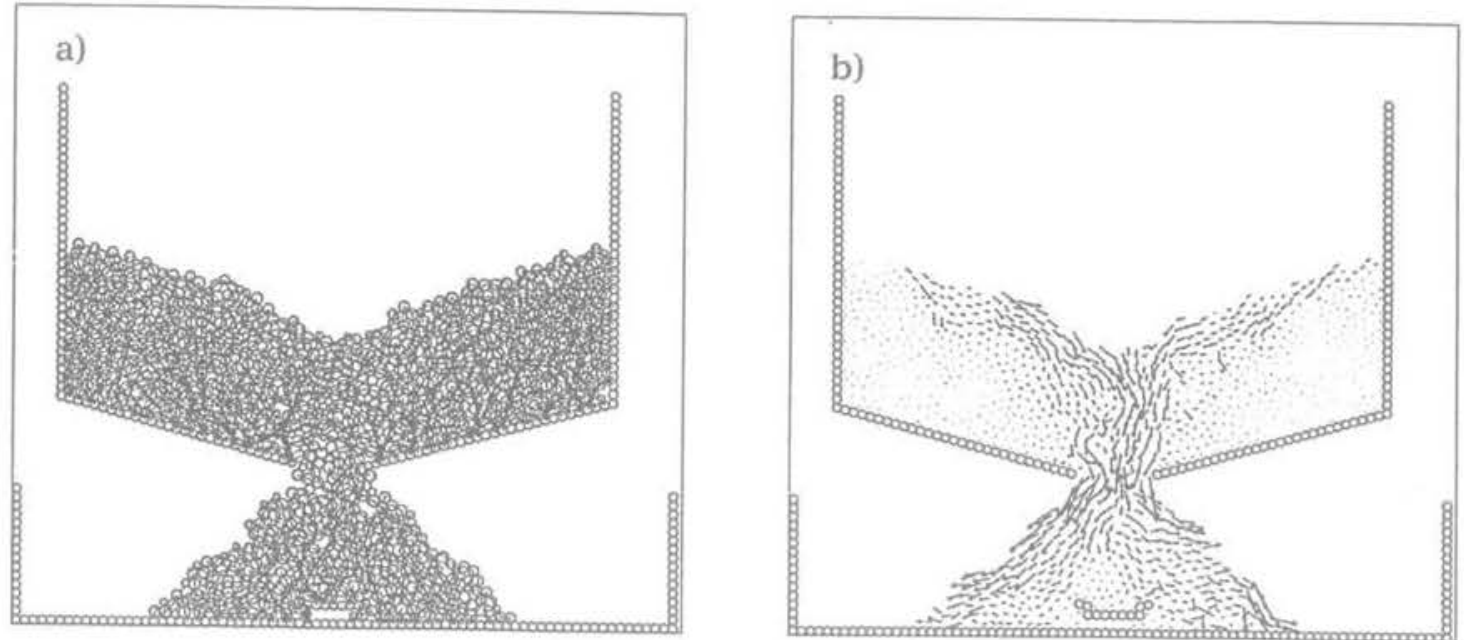

FiG. 21 Écoulement libre à travers l'ouverture (matériau sec, b/ $\mathrm{D}_{0}=8,33$ ), a) configuration intermédiaire au cours de la vidange, b) champ de vitesse des particules correspondant à l'état intermédiaire.

Free granular flow (dry materials, $b / D_{0}=8.33$ ), a) intermediate state configuration while emptying, b) particle velocity field corresponding to intermediate state.

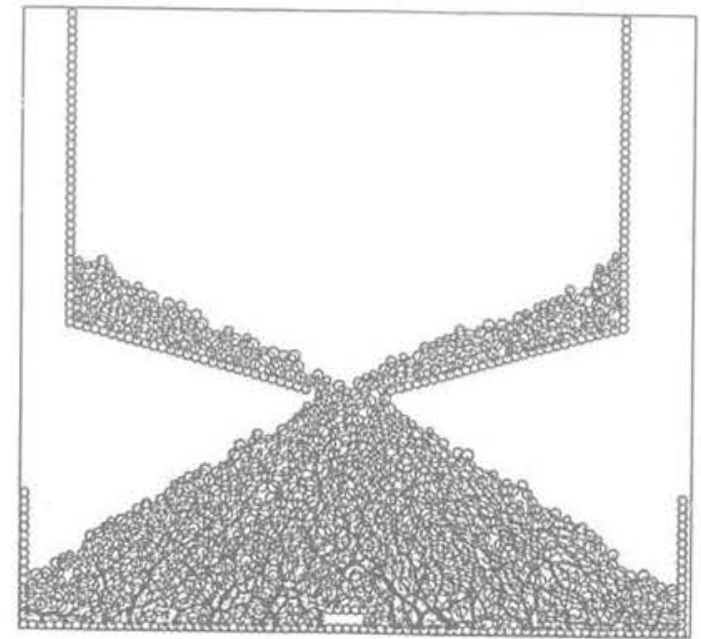

FIG.22 Configuration du silo et des grains à la fin de la vidange. (matériau sec, $\mathrm{b} / \mathrm{D}_{0}=8,33$ ). Final configuration of particles at the end of emptying (dry materials, $\left.\mathrm{b} / \mathrm{D}_{0}=4\right)$. 

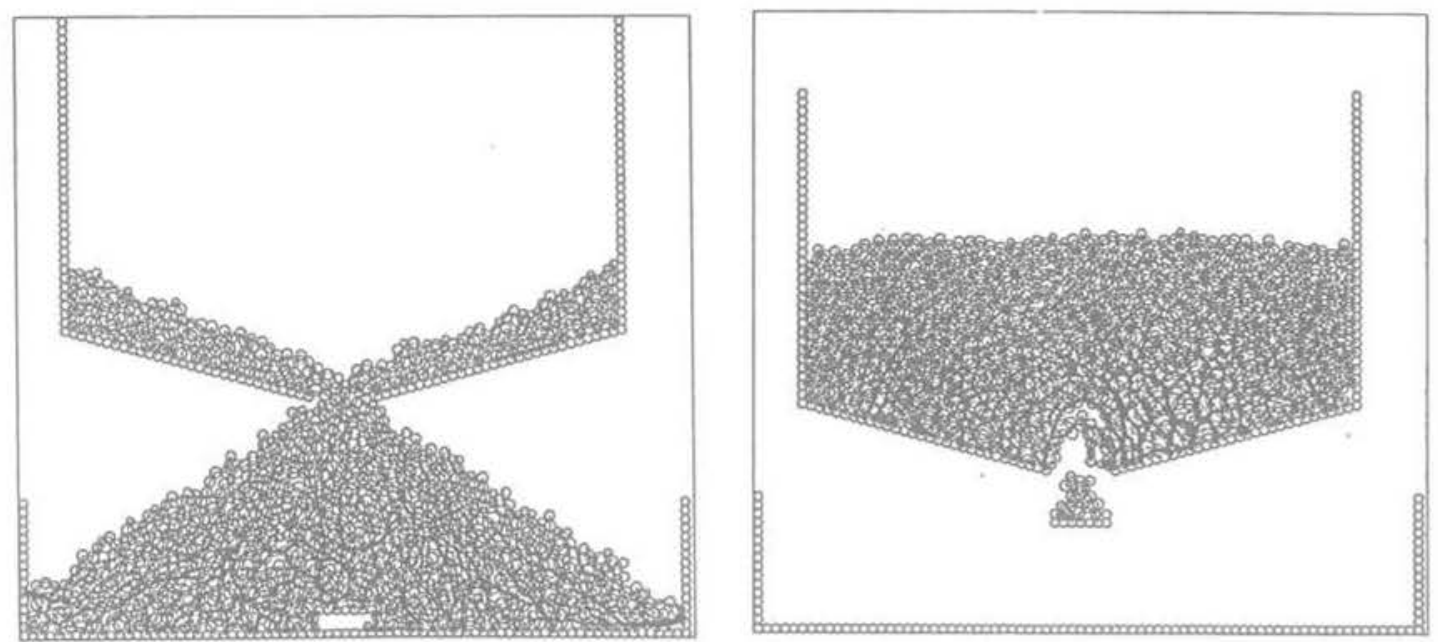

FiG. 23 Écoulement dépendant de la teneur en eau du matêriau $\left(\mathrm{b} / \mathrm{D}_{0}=6,50\right)$, a) matériau sec (écoulement libre), b) matériau humide (écoulement bloqué).

Granular flow depending on water content $\left(\mathrm{b} / \mathrm{D}_{0}=6.50\right)$, a) dry materials, free flow, b) wet material, blocked flow.

forme et bloque l'écoulement, comme le montre la figure 23. Il existe donc une ouverture minimale correspondant à une quantité d'eau donnée pour laquelle l'écoulement ne se produit pas. Les essais montrent que l'écoulement dans un silo peut être influencé par la cohésion résultant d'une très faible teneur en eau du matériau ensilé, et par la dimension de l'ouverture de décharge. L'ensemble de ces phénomènes est évidemment essentiellement lié à la nature discrète du milieu et ne pourrait être simulé à partir d'une approche basée sur la mécanique des milieux continus.

\section{7}

\section{Conclusion}

Le comportement des matériaux granulaires est souvent décrit à partir des modèles basés sur la théorie des milieux continus. Néanmoins certains résultats expérimentaux (ex. dilatance, quasi non-existence d'un domaine élastique, anisotropie induite) ne peuvent s'expliquer qu'à partir des phénomènes liés à la microstructure non-continue.

La méthode des éléments discrets est une méthode qui permet de prendre en compte la nature discontinue des milieux granulaires.

Les simulations effectuées sur un assemblage comportant 1000 particules bidimensionnelles montrent que le comportement simulé est représentatif et tous les aspects importants du comportement des matériaux granulaires, tel que l'influence de la compacité initiale et l'apparition de l'état critique pour des déformations importantes, sont mis en évidence. Les résultats locaux donnent une idée claire sur l'évolution de l'anisotropie dans le milieu granulaire. Ils montrent que l'anisotropie géométrique et statique se développent dans la direction de la contrainte majeure et se stabilisent avec la stabilisation de la contrainte déviatoire. L'influence du frottement local sur le frottement interne du milieu a été étudiée. Il montre que le frottement local a une influence sur le frottement interne au pic mais son influence sur le frottement interne au palier apparait faible.

Les simulations tridimensionnelles ont été réalisées sur un assemblage de 1000 particules sphériques. Malgré le nombre très réduit de particules, il semble que le comportement de l'assemblage granulaire soit correctement simulé. Les simulations d'essais effectués sur différents chemins des sollicitations ont permis d'obtenir le critère de rupture pour cet assemblage. Le critère ainsi obtenu est très proche des critères habituellement utilisés dans la modélisation élastoplastique du comportement des matériaux granulaires. L'influence de Thistoire des sollicitations a été numériquement mis en évidence, et l'évolution des surfaces de charge due à l'évolution de l'anisotropie induite est qualitativement comparable aux résultats expérimentaux obtenus par Lanier (1988).

Les simulations de stockage et d'écoulement de matériaux granulaires réalisés avec la MED ont montré une des applications possibles de cette méthode. Elles ont, en particulier, permis de caractériser la cinématique de l'écoulement, et de mettre en évidence l'influence de l'humidité dans le milieu et de la dimension de l'ouverture de vidange. La MED paraît particulièrement bien adaptée pour traiter ce type de problèmes d'écoulement pour lesquels les méthodes de la mécanique des milieux continus paraissent bien limitées. 
Abriak N.E., Mahboubi A. - Influence du frottement local sur le frottement global, Rapport annuel du Greco-Géomatériaux, France, 1993.

Bardet J.P. Proubet J. - A numerical investigation of the structure of persistent shear bands in granular media, Geotechnique, $41, n^{\circ}$ 4. 1991, p. 599-613.

Bathrust R.J., Rothenburg L. - Micromechanical aspects of isotropic granular assemblies with linear contact interaction, J. Appl. Mech., 55, 1988, p. 17-23.

Bathrust R.J., Rothenburg L. - Observations on stress-force-fabric relationships in idealized granular materials, J. Mechanics of Materials, 9, 1990, p. 65-80.

Cambou B., Jafari K. - Modèle de comportement des sols non cohérents, Revue française de géotechnique, $n^{\circ} 44,1988, p$. 43-55.

Cambon B. Lanier J. - Induced Anisotropy in Cohesionelss Soils, Experiments and Modelling, Computers and Geotechnique, $n^{\circ}$ 6. 1988, p. 291-311.

Chen Y., Hwang G. - Studies of the behavior of granular material and its fabric evolution, in Powders and Grains 93, ed. C. Thornston, Balkema, Rotterdam. 1993, p. $135-140$.

Cundall P.A. - A computer model for simulating progressive, large-scale movements in block rock systems, Proc. Symp, Int. Soc. Rock Mech., Nancy II, Art. 8, 1971.

Cundall P.A. - A computer model for rockmass behavior using interaction graphics for the inpunt and outpout of geometrical data, Report $n^{\circ}$ DACW 45-74-C-006, Missouri River Division. US Army Corps of Engineers, University of Minnosota, 1974.
Cundall P.A. - Computer simulation of dense sphere assemblies, in Micromechanics of granular material, ed. M. Satake \& J.T. Jenkins, Elsevier, Amsterdam. 1988, p. 113-123.

Cundall P.A. - Numerical experiments on localization in frictional materials, Ingenieur-Archiv, 59, 1989, p. 148-159,

Cundall P.A. Drescher A., Strack O.D.L. Numerical experiments on granular assemblies; measurements and observations, Proc. of IUTAM conf. on Deformation and failure of granular materials, Delft, 1982, p. 355-370.

Cundall P.A.. Strack O.D.L. - A discrete numerical model for granular assemblies, Geotechnique, $29, n^{\circ} 1,1979$, p. 47 65

Desrues J. - Strain localization and interfaces: research needs in France, in Proc. joint US-France worshop in recent advances in Geomechanics, Geotechnical and Geo-envirommental Engineering, ed. F Darve, Y. Meimon, J. Benoit, R.H. Borden, Technip. Paris, 1993, p. 47-58.

Hertz H. - Uber die Beruhrung fester elastischer Korper, J. F. reine und angew Mathematik (Creeles J.), vol. 92, 1981.

Jean $\mathrm{M}$. - Simulation numérique des problèmes de contact avec frottement, Journées de la Société Tribologique de France, Sophia-Antipolis, 9-10 avril 1992, Matériaux et Techniques, $n^{\circ} 1-2-3$, 1993.

Kruyt N.P. - Toward micro mechanical constitutive relations for granular materials based on two dimensional discrete element simulation, in Modern approches to plasticity, ed. D. Kolymbas, Elsevier. 1993.
Lade V., Duncan M. - Elastoplastic stress strain theory for cohesionless soil, Proc: ASCE 101, GT10; 1975, p. 1037-1053.

Love A.E.H. - A treatise of mathematical theory of elasticity, University Press, Cambridge, 1927.

Mahboubi A. - Contribution à l'étude micromécanique du comportement des matériaux granulaires par homogénéisation et approche numérique, Thèse de Doctorat, Ecole Centrale de Lyon, France, 1995

Mindlin R.D., Derisiewicz H. - Elastic spheres in contact under varying oblique forces, Trans. ASME, $J$ Applied Mech, 20, 1953, p. 327-344.

Moreau J.J. - Application of convex analysis to some problems of dry friction, in Trends in application of pure mathematics to mechanics, ed. H. Zorski, Vol. 2. Pitman. London, 1979, p. 263-280.

Moreau J.J. - Unilater contact and dry fiction in finite freedom dynamics, CISM Courses and Lectures, $n^{\circ} 302$, SpringerVerlag, Wien, 1988, p. 1-82.

Ng T.T. Dorby R. - Numerical simulation of monotonic and cyclic loading of granular soil, J, of Geotechnical Eng., vol. $120, n^{\circ} 2,1994$, p. 388-403.

Rothenburg L., Bathrust R.J. - Analytical study of Induced Anisotropy in Idealized Granular Materials, Geotechnique, 39, n 4, 1989, p. 601-614.

Skinner A.E. - A note on the influence of interparticle friction. Geotechnique, 19 , 1969, p. 150-157.

Thornton C., Barnes D.J. - Computer simulated deformation of compacted granular assemblies, Acta Mechanica, 64,1986, p. $45-61$ 